\title{
The evolutionary young miR-1290 favors mitotic exit and differentiation of human neural progenitors through altering the cell cycle proteins
}

\author{
SV Yelamanchili, ${ }^{\star, 1}$ B Morsey ${ }^{1}$, EB Harrison ${ }^{1}$, DA Rennard ${ }^{1}$, K Emanuel $^{1}$, I Thapa ${ }^{2}$, DR Bastola ${ }^{2}$ and HS Fox ${ }^{\star, 1}$
}

Regulation of cellular proliferation and differentiation during brain development results from processes requiring several regulatory networks to function in synchrony. MicroRNAs are part of this regulatory system. Although many microRNAs are evolutionarily conserved, recent evolution of such regulatory molecules can enable the acquisition of new means of attaining specialized functions. Here we identify and report the novel expression and functions of a human and higher primate-specific microRNA, miR-1290, in neurons. Using human fetal-derived neural progenitors, SH-SY5Y neuroblastoma cell line and H9-ESCderived neural progenitors (H9-NPC), we found miR-1290 to be upregulated during neuronal differentiation, using microarray, northern blotting and qRT-PCR. We then conducted knockdown and overexpression experiments to look at the functional consequences of perturbed miR-1290 levels. Knockdown of miR-1290 inhibited differentiation and induced proliferation in differentiated neurons; correspondingly, miR-1290 overexpression in progenitors led to a slowing down of the cell cycle and differentiation to neuronal phenotypes. Consequently, we identified that crucial cell cycle proteins were aberrantly changed in expression level. Therefore, we conclude that miR-1290 is required for maintaining neurons in a differentiated state.

Cell Death and Disease (2014) 5, e982; doi:10.1038/cddis.2013.498; published online 9 January 2014

Subject Category: Neuroscience

The brain is a unique and complex structure. Primates, particularly apes and humans, have brains that are clearly distinct both morphologically and functionally from the rest of the animal kingdom. This evolutionary process is accompanied by tightly regulated interplay of cellular and molecular processes. The molecular events that differ during the development of higher-order animals remain largely unknown. An important event in development, the expansion of neural progenitor reservoirs and their differentiation to generate specific neuronal subtypes, is under the control of several extrinsic and intrinsic factors. Recently, small noncoding RNA molecules, microRNAs (miRNAs) have been shown to play a pivotal role in embryonic development ${ }^{1,2}$ and have remained attractive candidates to study properties of neural stem cells such as neurogenesis and neural fate commitment. ${ }^{3}$ Global disruption of miRNAs through ablation of Dicer in neural stem cells lead to significant defects in embryonic mouse brain development. ${ }^{4,5}$ Importantly, certain miRNAs affect neuronal differentiation via regulation of their mRNA targets. ${ }^{6-8}$

The majority of studies on miRNAs and the brain have been carried out on miRNAs that are conserved during evolution, and the conservation of miRNA target in the mRNA $3^{\prime}$-untranslated region (UTR) is used as a criterion in assessing potential miRNA actions on a gene. ${ }^{9}$ Recently, a number of evolutionarily young miRNAs have been found. ${ }^{10-12}$ Many of the novel miRNAs discovered in higher species are differentially expressed in developing tissues, supporting the notion that miRNAs may have a key role in the evolved complexity of higher mammals. ${ }^{11}$ However, the role of these nonconserved miRNAs is yet not clear, largely because of the limitations that accompany studying higher organisms and organs such as the brain. Here we investigated the function of a higher species miRNA in human neuronal development through the use of three different model systems. We have identified miR-1290, found only in great apes (including humans), as an important regulator of human neuronal differentiation process by regulating the levels of critical cell cycle proteins.

\section{Results}

Identification and validation of higher species-specific miR-1290 in neurons. Isolated single cells from aborted human fetal brains were grown as neurospheres for a week and then further differentiated into postmitotic neurons in culture for 21 days in vitro (DIV) (Supplementary Figure 1A). Positive immunostaining for markers such as Nestin and

\footnotetext{
${ }^{1}$ Department of Pharmacology and Experimental Neuroscience, University of Nebraska Medical Center, Omaha, NE, USA and ${ }^{2}$ School of Interdisciplinary Informatics, University of Nebraska-Omaha, Omaha, NE, USA

${ }^{*}$ Corresponding author: SV Yelamanchili or HS Fox, Department of Pharmacology and Experimental Neuroscience, University of Nebraska Medical Center, 985800 Nebraska Medical Center-DRC 3008, Omaha, NE 68198-5800, USA. Tel: +1 402559 4821; Fax: + 1402559 7495; E-mail:syelamanchili@unmc.edu or hfox@unmc.edu

Keywords: microRNA; neural differentiation; neural progenitor cell; cell cycle

Abbreviations: miRNA, microRNA; UTR, untranslated region; hNPC, human neural progenitor cell; hNPC-N, human neural progenitor cell-derived neuron; hNPC-A, human neural progenitor cell-derived astrocyte; ISH, in situ hybridization; BrdU, bromodeoxyuridine; TUNEL, terminal deoxynucleotidyl transferase dUTP nick end labeling

Received 05.7.13; revised 18.10.13; accepted 05.11.13; Edited by A Verkhratsky
} 
SOX2 as well as PAX6 and Ki67 (Supplementary Figure 1B) indicated the progenitor identity and proliferative capacity of the human neural progenitor cells (hNPCs). Upon differentiation, populations enriched for neurons (hNPC-Ns) were produced. These neuronal cultures expressed markers such as MAP2, synaptic vesicle marker VGLUT1 and immature neuronal marker TUC-4. In addition, a minority of cells in these cultures also expressed the astrocytic marker GFAP (Supplementary Figure 1C).

To assess alterations in miRNAs, RNA isolated from hNPCs and hNPC-Ns from three independent donors was hybridized to Affymetrix miRNA microarray chips (Santa Clara, CA, USA). Several miRNAs were differentially expressed (Supplementary Table 1), and the greatest fold change and significance in hNPC-Ns when compared with progenitor cells was for miR-1290 (Figure 1a, left panel). Further validation by quantitative real-time PCR (qRT-PCR) confirmed a significant increase in miR-1290 expression (15-fold, $P<0.001, n=3$, $t$-test) in hNPC-Ns compared with hNPCs (Figure 1a, right panel). To validate this finding we next performed northern blotting on RNA derived from hNPC and hNPC-N samples on four independent donors. The northern blot revealed the expression of the mature form $(\sim 21 \mathrm{nt})$ in hNPC-N but not in hNPC samples (Figure 1b). Next, we asked whether miR-1290 expression is associated with neuronal, as opposed to astrocytic, differentiation. The hNPCs containing neurospheres were therefore differentiated to either astrocytes (hNPC-As) or neurons (hNPC-Ns) (Figure 1c). qRT-PCR from three independent donors indicated a robust signal from neurons relative to a low baseline in astrocytes ( $>100$-fold, $P<0.001, n=3, t$-test), indicating that miR-1290 is strongly associated with a neuronal phenotype.

MiR-1290 is encoded in the first intron of the $A L D H 4 A 1$ gene in the human genome, and its expression has only been described in humans. We examined the Multiz sequence alignment ${ }^{13}$ of 44 vertebrate genomic sequences to assess the evolution of miR-1290. Our results indicate that miR-1290 homologs are present in the Haplorhini clade of primates but not other vertebrates, dating its origin to $\sim 87.2$ million years ago $^{14}$ and that the mature miR-1290 sequence is exclusive to the subfamily Homininae (the great apes including humans), dating its origin to 16.5 million years ago ${ }^{14}$ (Supplementary Figure 2).

Although several sequencing studies have reported the identification of mature miR-1290, ${ }^{15-17}$ we validated its ability to be expressed experimentally by the introduction of a $31.4 \mathrm{~kb}$ fosmid clone containing the ALDH4A1 and MIR1290 genomic region from humans into mouse NIH3T3 cells that otherwise do not express miR-1290, lacking the sequence in the genome. The in situ hybridization (ISH) revealed the expression of miR-1290 in only transfected cells (Supplementary Figure 3), and qRT-PCR corroborated expression of miR-1290 in transfected cells but not mocktransfected cells (>100-fold, $P<0.001, \quad n=3, \quad t$-test), confirming its ability to be expressed and processed.

Next, we performed ISH on human fetal (20-week gestation) and adult brain sections to assess the expression of miR-1290. ISH on the fetal frontal cortical sections revealed that miR-1290 expression was highly nuclear. In order to eliminate the possibility of false-positive signals that may arise because of nonspecific binding of the probe to the genomic DNA in the nucleus, we treated the fetal sections with RNAse A that completely destroyed both the positive control U6 signal and miR-1290 from the nucleus, indicating that the signal was indeed because of RNA and may result from the unprocessed pri- and pre-forms (Figure 2a, upper panels). In adult human frontal brain sections, miR-1290 was highly expressed in cytoplasm rather than in the nucleus, consistent with the mature form (Figure 2b, lower panels).

The miR-1290 expression in differentiating human neuronal cells. In order to model neuronal differentiation in vitro, we then performed a time course analysis of differentiating primary hNPCs. ISH indicated a steady increase in miR-1290 during the course of differentiation along with the neuronal differentiation marker Tuj1 (Figure 3a). Simultaneously performed qRT-PCR analysis corroborated with the results, thereby associating increase in miR-1290 expression with neuronal differentiation (Figure $3 \mathrm{~b}$, left panel).

Next, we utilized the neuroblastoma cell line SH-SY5Y that can be induced to differentiate into neurons in culture to model the differentiation process. Indeed, such neuronal differentiation of SH-SY5Y cells led to a significant upregulation of miR1290 expression (Figure 3b, right panel).

Finally, we differentiated the $\mathrm{H} 9$ human embryonic stem cell line (H9-hESC)-derived NPCs (H9-NPCs). H9-NPCs were positive for neural progenitor markers such as Nestin, Sox2 and Pax6, and for proliferative markers such as Mushashi-1 (MUSH-1). They were negative for the embryonic stem cell marker OCT4, indicating commitment to neural lineage as well as for postmitotic marker TUC-4 (Supplementary Figure 4). Upon differentiation to neurons for 7 DIV, these cells expressed all the neuronal markers examined: MAP2, NeuN and Tuj-1 (Figure 3c), along with an increase in miR-1290 expression in phenotypic neurons (Figure 3c, bottom panels). Furthermore, qRT-PCR revealed a significant increase in miR-1290 expression in day 7 differentiated neurons, corroborating with our initial observation in primary cells (threefold, $P<0.001, n=4, t$-test; Figure $3 c$, right). These data further ascertain that increased miR-1290 expression is strongly associated with neuronal differentiation process.

The miR-1290 inhibition in differentiated immature neurons leads to increased cellular proliferation and eventually cell death. As postmitotic differentiation process increased the expression of miR-1290 drastically, we assessed the effect of inhibition of miR-1290 in such cells. H9-NPCs were transfected with miR-1290 or negative (Neg) inhibitors followed by neuronal differentiation as described previously. Inhibition of miR-1290 during differentiation of progenitor cells showed a dramatic increase in cellular proliferation that is indicated by bromodeoxyuridine (BrdU) labeling as well as Ki67 staining when compared with controls (Figures $4 a$ and $b$ ); the lower proportion of BrdU or Ki67-positive cells in the negative control cultures might represent newly born neurons. In addition, we assessed the proliferative growth by determining the density of cultures 


$\begin{array}{ccccc}\text { a Column \# } & \text { Column ID } & \text { p-value } & \text { Ratio (log2) } & \text { Fold Change } \\ 3193 & \text { hsa-miR-1290_st } & 1.16 \mathrm{E}-03 & 3.89 & 14.79 \\ 3133 & \text { hsa-miR-1246_st } & 1.62 \mathrm{E}-02 & 3.27 & 9.66 \\ 3319 & \text { hsa-miR-195-star_st } & 2.60 \mathrm{E}-02 & 2.59 & 6.04 \\ 3405 & \text { hsa-miR-297_st } & 4.38 \mathrm{E}-02 & 2.45 & 5.45 \\ 3762 & \text { hsa-miR-610_st } & 1.41 \mathrm{E}-02 & 2.17 & 4.51 \\ 3295 & \text { hsa-miR-185-star_st } & 1.49 \mathrm{E}-01 & 1.72 & 3.30\end{array}$
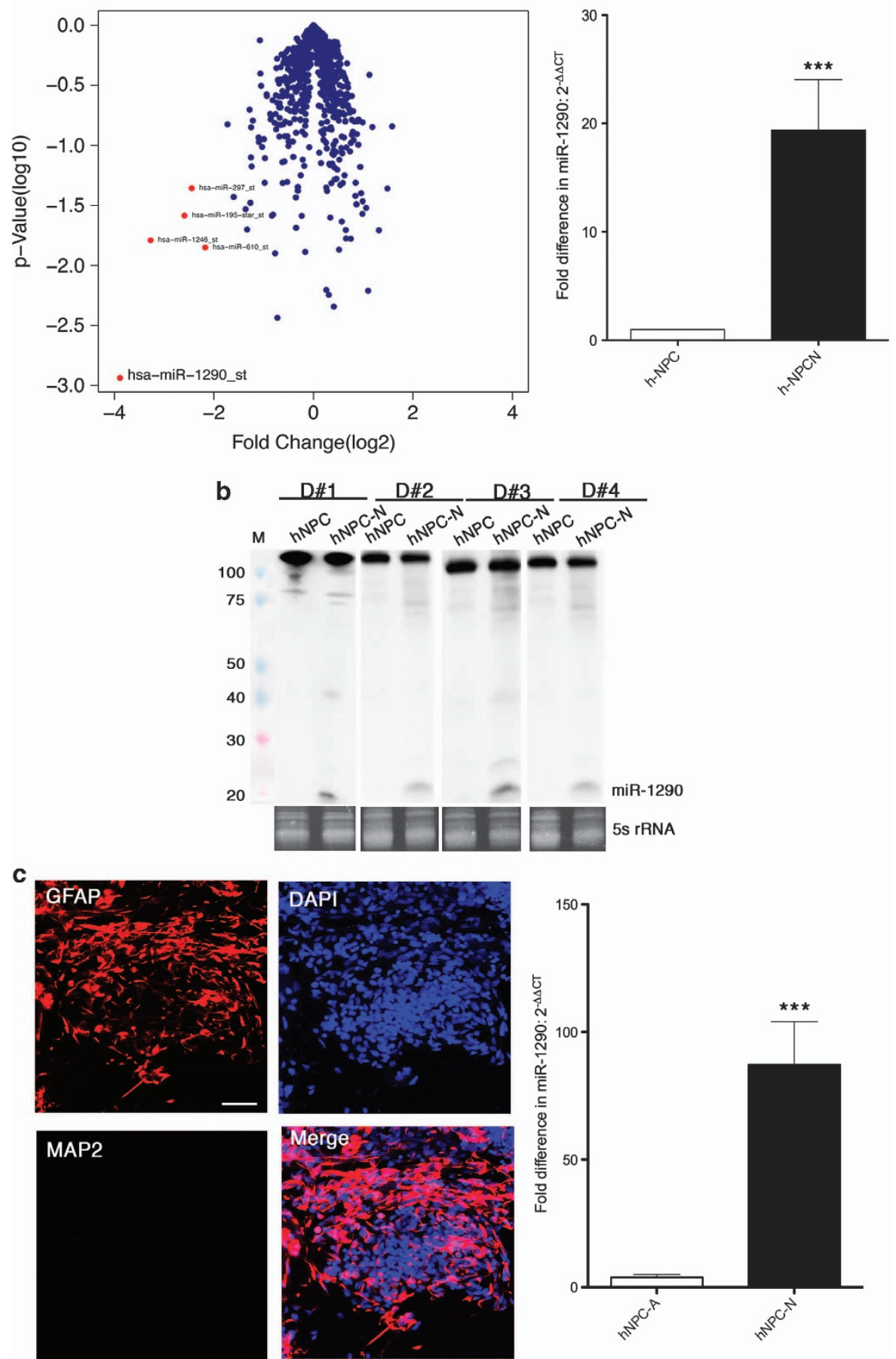

Figure 1 MiR-1290 is upregulated during neuronal differentiation. (a) Top five miRNAs that were significantly upregulated in neurons. Volcano plot of the miRNA microarray performed on three independent donors shows an $\sim 15$-fold $(P<0.001$, unpaired Student's $t$-test) expression of miR-1290 in h-NPCNs when compared with h-NPCs. Bar graph on the right represents the postvalidation of miR-1290 by qRT-PCR, and results indicate a 15 -fold $(P<0.001, n=3)$ increase in miR-1290 expression in hNPCNs. (b) Representative northern blot of RNA derived from hNPCs and hNPC-Ns from four individual donors (D1-D4); note that the anti-DIG signals for the mature form ( $21 \mathrm{nt})$ were only seen after neuronal differentiation (hNPC-N). A prestained small molecular weight miRNA marker (M) was used to monitor RNA size. (c) Double immunostaining of astrocytic marker GFAP and neuronal marker MAP2 performed on hNPC differentiated astrocytes in culture, bar $=20 \mu \mathrm{m}$. Bar graph on the right represents 104-fold increased expression in miR-1290 in h-NPC-Ns when compared with astrocytes (104-fold, ${ }^{* \star *} P<0.001, n=3$, unpaired Student's $t$-test) 
a

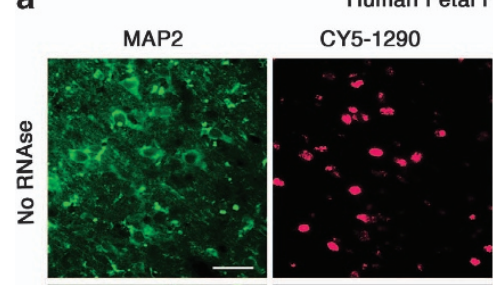

DAPI
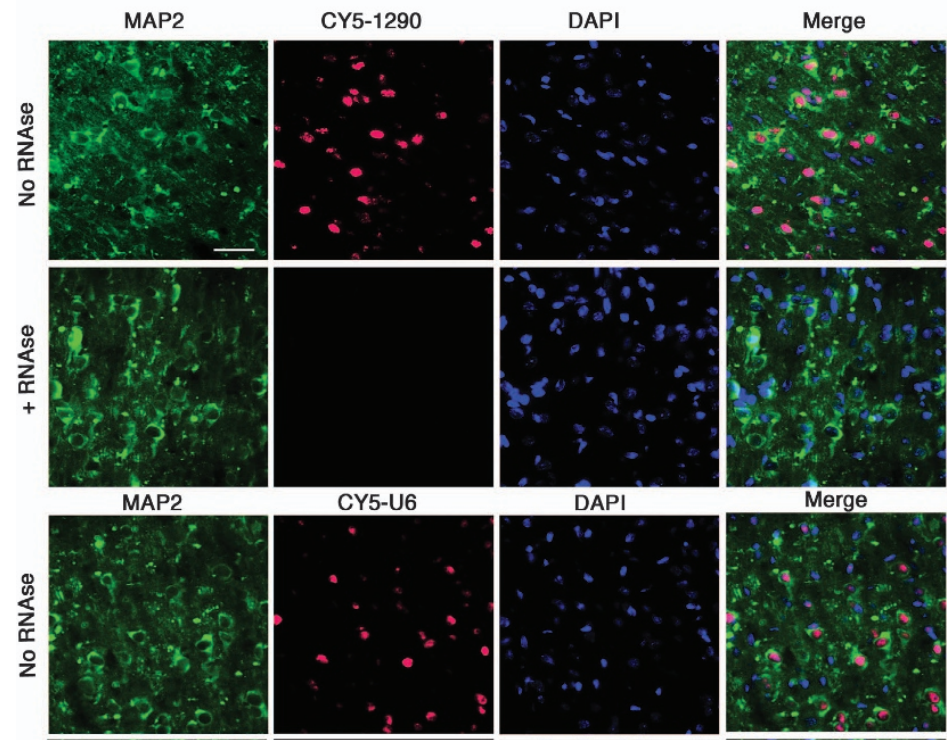

CY5-U6

DAPI
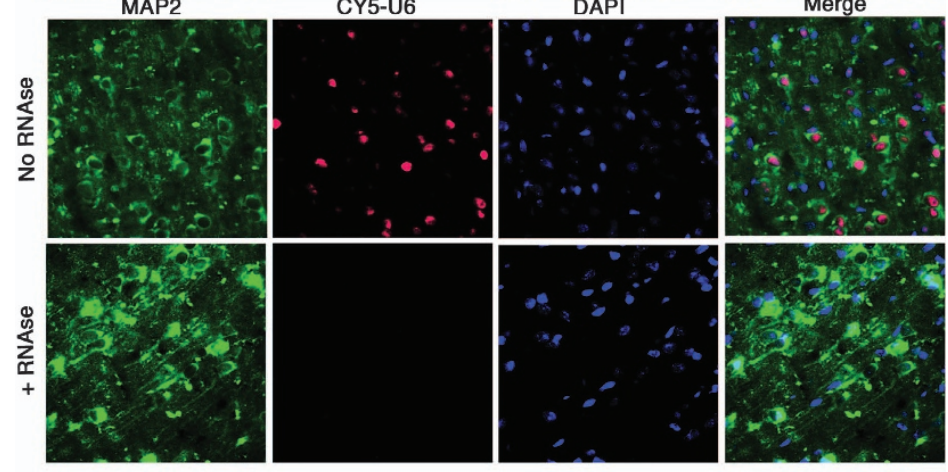

b

b MAP2
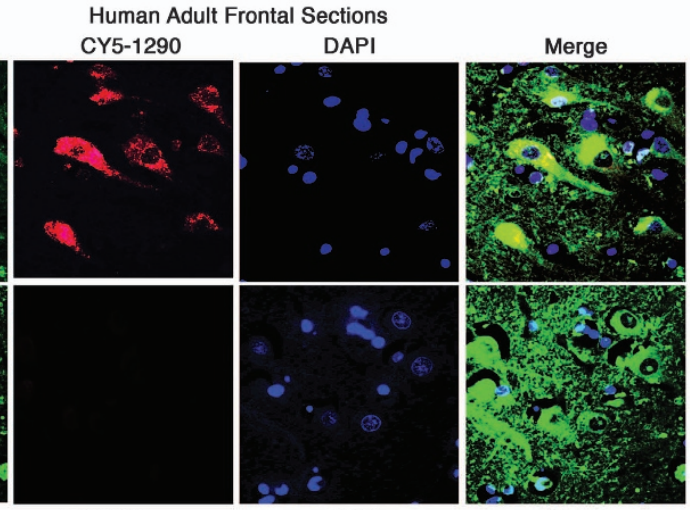

MAP2

CY5-U6

DAPI

Merge
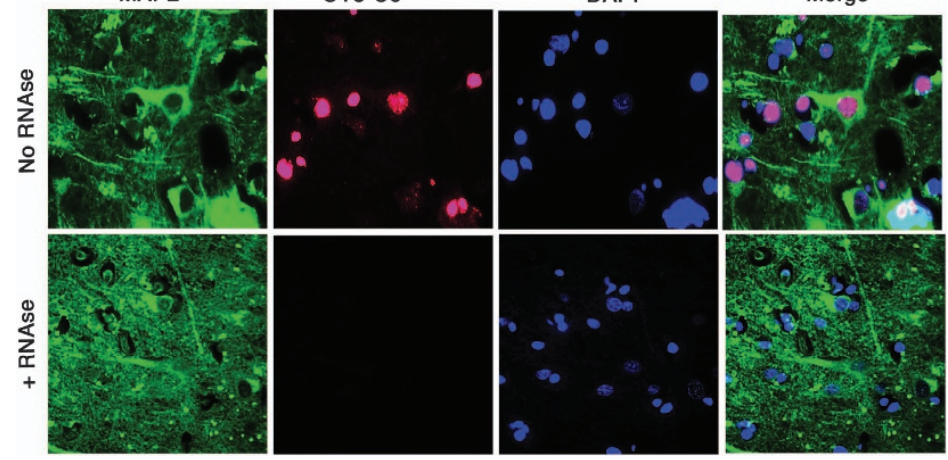

Figure 2 MiR-1290 signal localizes to nucleus in fetal neurons and to cytoplasm in adult neurons. (a) Double immunolabeling (MAP2) in situ hybridization was performed on human fetal frontal brain sections with CY5-labeled miR-1290 (upper panels) or a positive control U6 (lower panels). In addition, RNAse A treatment on sections before probe hybridization abolished any signal for both miR-1290 and U6, confirming that nuclear signals seen in fetal sections are not the result of nonspecific probe binding to genomic DNA. Bar $=20 \mu \mathrm{m}$. (b) Double immunolabeling (MAP2) coupled to in situ hybridization (Cy5-labeled miR-1290) on human adult frontal brain sections. MAP2/miR1290 staining is evident in MAP2-positive neurons where staining is mostly in the cytoplasm. Note that RNase A treatment before probe hybridization abolished miR-1290 or U6 signals from cytoplasm and nucleus respectively. Bar $=20 \mu \mathrm{m}$ 
grown on numeric grid coverslips and saw a drastic increase in density after miR-1290 inhibition (Figure 4c). We hypothesized that such miR-1290 inhibition-induced proliferation in differentiating cells could lead to apoptosis, and therefore performed terminal deoxynucleotidyl transferase dUTP nick end labeling (TUNEL) staining. We observed an increase in TUNEL-positive cells, confirming that inhibiting miR-1290 not only disrupted the normal differentiation process but also led to untimely cell death (Figure 4d). To further examine the effects on cell proliferation, we transfected SH-SY5Y cells grown in differentiation medium with miR-1290 inhibitor. The miR-1290 inhibitor-transfected cells divided more rapidly, forming a cluster or a colony by day 3 followed by increased proliferation by day 6 . On the other hand, the negative control inhibitor-transfected cell revealed normal differentiation process (Figure 4e).

Finally, we examined the effect of miR-1290 inhibition in differentiated primary human neurons. We transfected neurons in culture at DIV 21 (where the highest peak of miR-1290 expression was seen) with miR-1290 inhibitor or a negative control sequence. ISH revealed the loss of miR-1290 in the neurons that were transfected with the miR-1290 inhibitor when compared with the negative control (Supplementary Figure 5). During 5 days in culture with the inhibitor, as compared with the no inhibitor and negative controls, the miR-1290-inhibited cells started to lose their processes and formed colonies, indicating an increase in proliferation, as measured by BrdU labeling (Figure 5). Results from two different neuronal differentiation models clearly indicate that inhibiting miR-1290 not only disrupted differentiation, but also increased proliferation, and ultimately led to apoptotic cell death.

The miR-1290 overexpression in undifferentiated cells results in slowing down of the cell cycle and induces neuronal differentiation. Next, we asked whether we could induce differentiation by overexpressing miR-1290 in neural progenitor cells. Using a lentiviral vector to overexpress miR-1290 (and coexpress fluorescent dsRED) in undifferentiated SH-SY5Y cells, cell cycle analysis performed using flow cytometry revealed a decrease in proliferating cells, with a higher proportion of cells in G0/G1 (Figure 6a, control lentivirus $60.17 \pm 0.95$, miR-1290 lentivirus $83.33 \pm 0.67$, $P<0.001, n=6, t$-test) and lower proportion of cells in the $S$ phase as compared with control (Figure 6a, control lentivirus $22.33 \pm 0.80$, miR-1290 lentivirus $6.72 \pm 0.17$, $P<0.001, n=6$, $t$-test). We further validated this result in H9-NPCs by transducing them with lentivirus and selecting cells for puromycin resistance in medium containing growth factors that induce proliferation. We observed a dramatic decrease in colony size when transduced with miR-1290 (Figure 6b). We hypothesized that this could be because of exiting of cells from cell cycle in order to differentiate. Indeed, miR-1290-transduced H9-NPCs showed evidence of neuronal differentiation as indicated by Tuj1 staining (Figure 6c, control lentivirus $9.00 \pm 2.65$, miR-1290 lentivirus $56.67 \pm 2.40, P<0.001, n=3, \quad t$-test). In order to better characterize the miR-1290-overexpressing H9-NPCs, we performed more in-depth examination of Tuj1-positive cells as well as cells expressing an additional neuronal marker
Tuc-4 and the proliferative marker Ki67. The differentiation marker Tuj1 showed a stronger staining in cells with neuronal phenotype than the surrounding cells (Figure 7a, see white arrows). Similarly, miR-1290-overexpressing cells also stain strongly with postmitotic marker Tuc-4 (Figure 7b). Furthermore, compared with the cells transduced with the negative control lentivirus, Ki67 staining was completely lost in cells that overexpress miR-1290 (Figures 7c and d), confirming reduced cellular proliferation.

The miR-1290 targets key cell cycle proteins. The miR-1290 inhibition and overexpression experiments in different cell systems have confirmed our hypothesis that miR-1290 not only plays an important role in neural differentiation, but also that its lowered expression is necessary to keep the cells in a proliferative stage, a critical process during development. Therefore, we hypothesized its role in maintaining cell cycle repression in neurons. Repression of gene expression by miRNAs is initiated by its recognition of target sequences, largely in the $3^{\prime}$-UTR of mRNAs. To determine the potential targets we examined the $3^{\prime}$-UTRs of all mRNA transcripts for complementarity to the miR-1290 seed region. These gene targets were examined for enrichment in Gene Ontology categories, and although many were present in functional categories related to brain developmental processes including neuron differentiation, neural fate commitment and neurogenesis, we also saw an significant enrichment of target genes whose proteins were involved in the categories of cell cycle, cell cycle arrest, G1/S transition of mitotic cell cycle and interphase of mitotic cell cycle (Supplementary Table 1). These targets included cyclins, cyclin kinases and cyclin kinase inhibitors. The effect of miR-1290 on cell cycle regulators was then examined by western blot analysis on samples derived from miR-1290 overexpression in undifferentiated H9-NPCs and miR-1290 inhibition in neuronally differentiated H9-NPCs. The important cyclin kinase inhibitor p27kip1 (p27), which is responsible for cell cycle exit, and its regulatory components CDK5 and $\mathrm{p} 35$, which positively regulate the production of $\mathrm{p} 27$, were examined. Results indicate that when miR-1290 is inhibited, p27 levels are significantly decreased along with CDK5 and p35, whereas proliferating cell nuclear antigen (PCNA) increased (Figure 8a), consistent with miR-1290 inhibition leading to proliferation. Conversely, overexpression of miR-1290 led to increase in p27, CDK5 and p35 levels and decrease in PCNA (Figure 8b), consistent with mIR-1290 expression leading to increased cell cycle exit. These data correlate well with our finding that overexpression of miR-1290 caused decrease in cell number or colony size.

\section{Discussion}

The differentiation of multipotent neural progenitors to neurons involves complex changes in gene expression that can be driven by transcriptional and post-transcriptional mechanisms. To learn more about these changes, we examined miRNAs, key post-transcriptional regulators of gene expression with effects on neuronal differentiation. Newly acquired miRNAs in genomes of higher species can alter the physiological gene balance at specific stages, for 
a Human fetal derived NPC

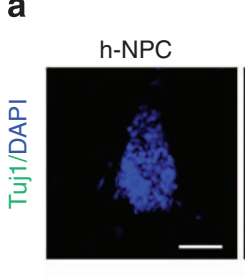

D1

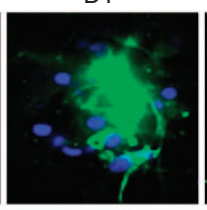

D3
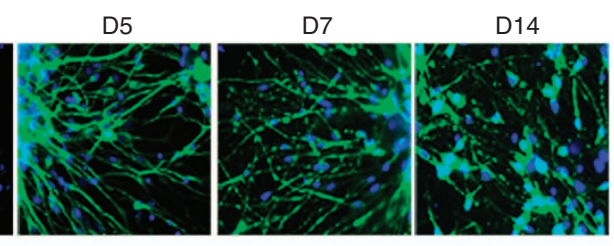

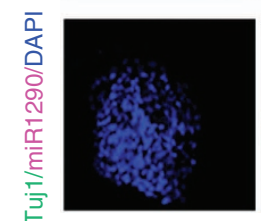
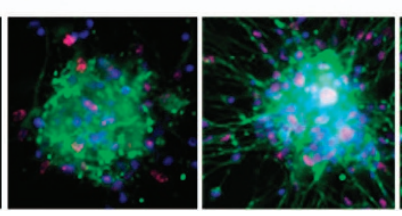

b
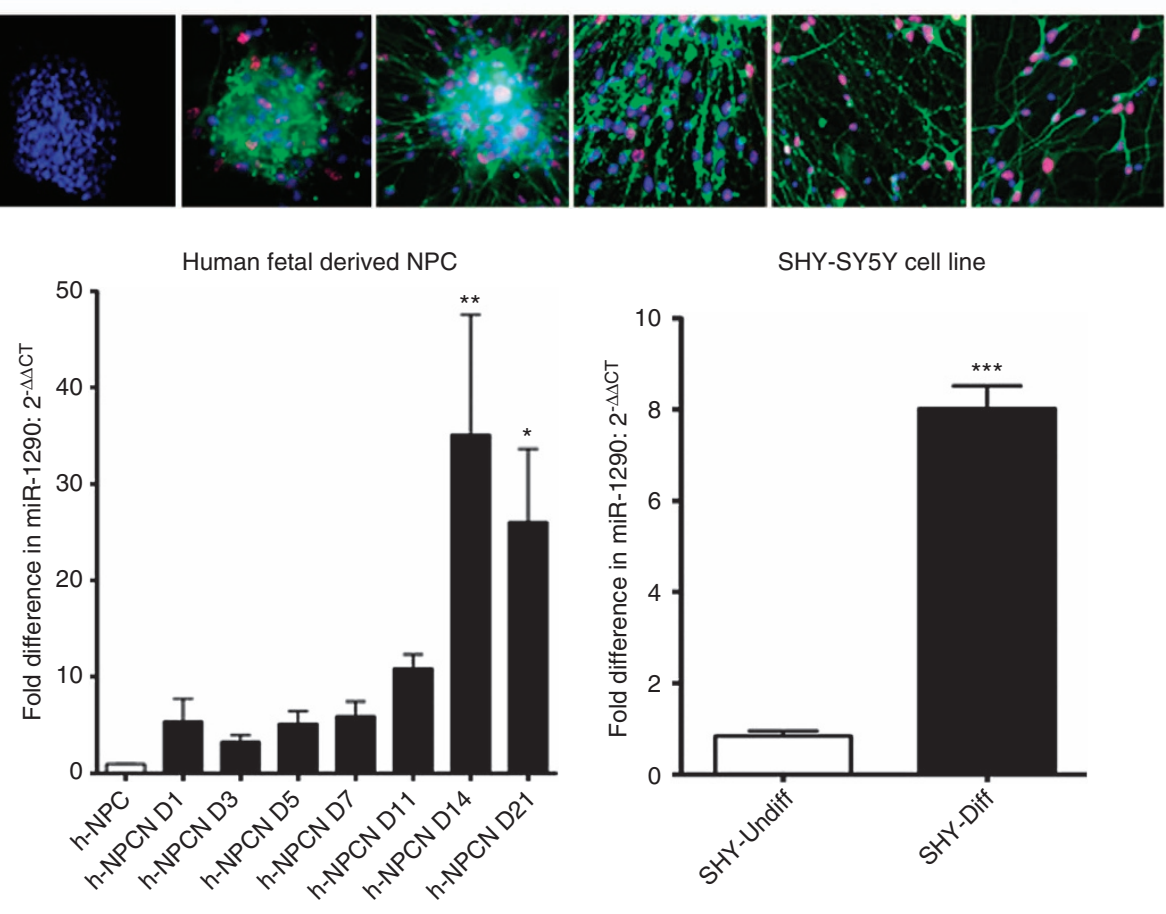

c

H9-NPC derived neurons

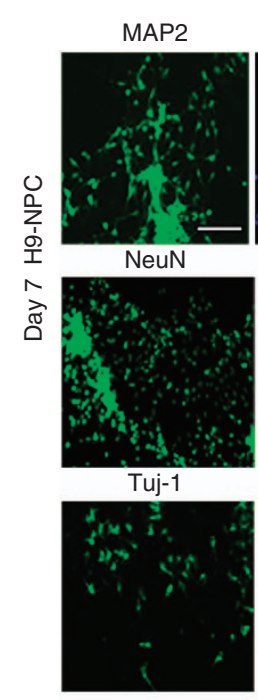

Day 1

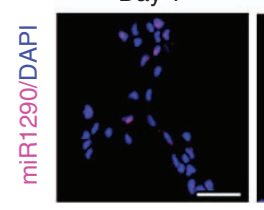

DAPI
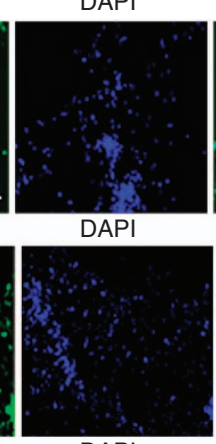

DAPI

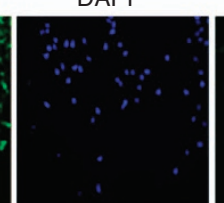

Day 3

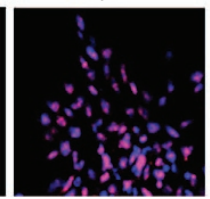

Merge
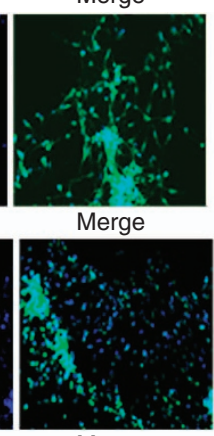

Merge

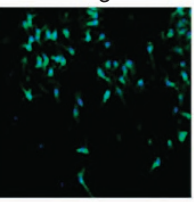

Day 7

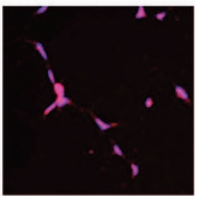

Day 7

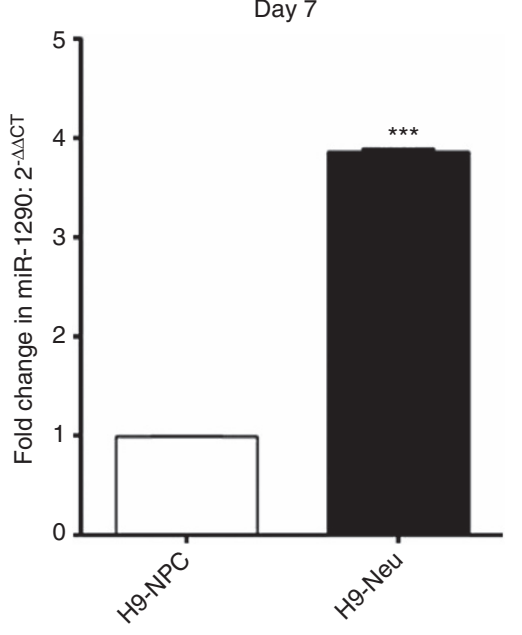


example during development. ${ }^{18}$ Our data are consistent with these findings. We identified miR-1290, an evolutionary young miRNA, as an important regulator of neuronal differentiation in newly born neurons. Inhibition of miR-1290 in differentiated H9-NPCs and human primary neurons reverted their postmitotic state, and prolonged inhibition of miR-1290 in differentiated cells led to cellular death. Overexpression of miR-1290 in undifferentiated cells led to slowing down of cell cycle in SH-SY5Y cells and led to differentiation to neurons in H9-NPCs. Furthermore, we showed that important regulators for cell cycle exit are perturbed such as p27kip1, CDK5 and p35 during knockdown or overexpression of miR-1290.

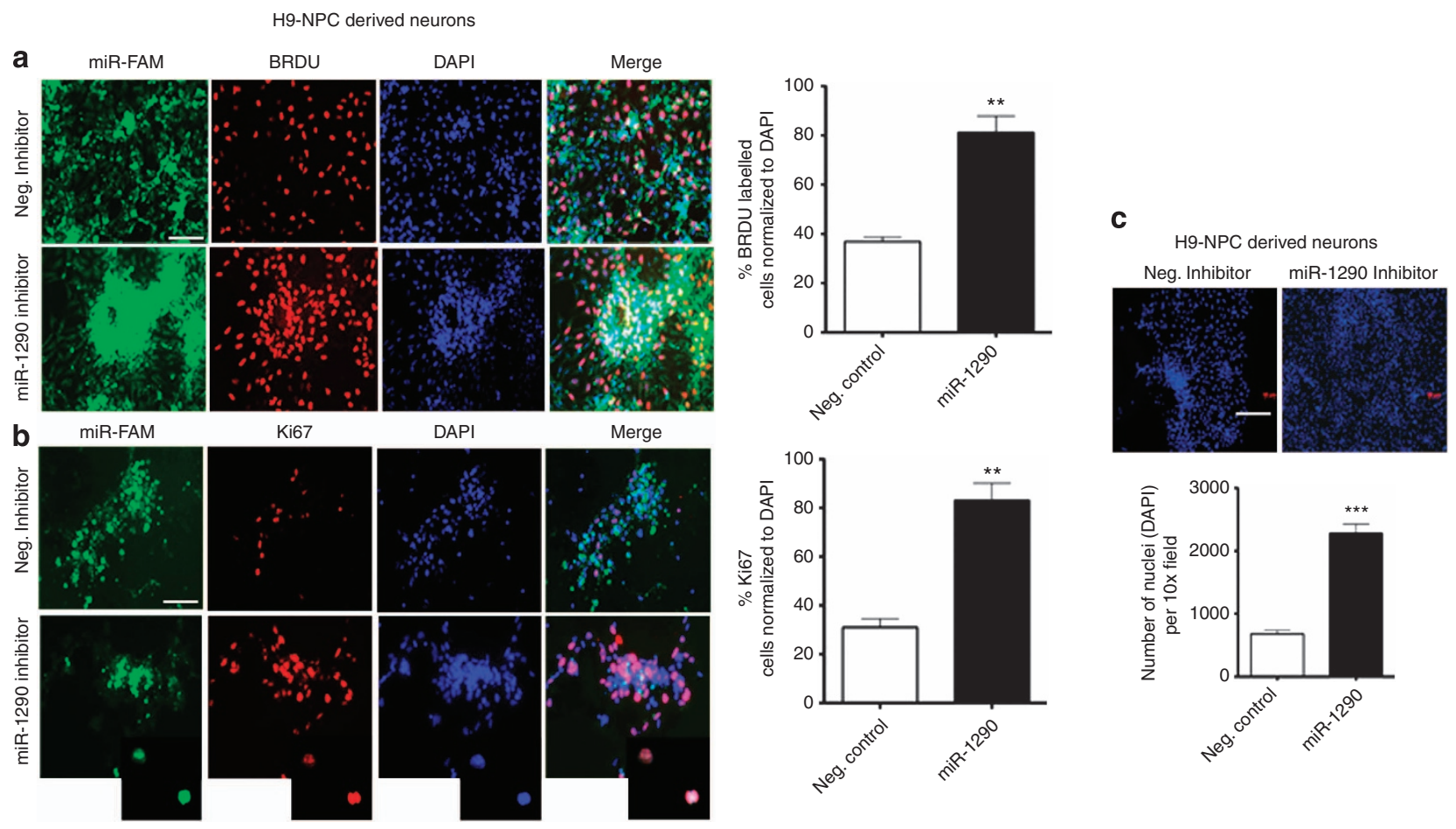

d

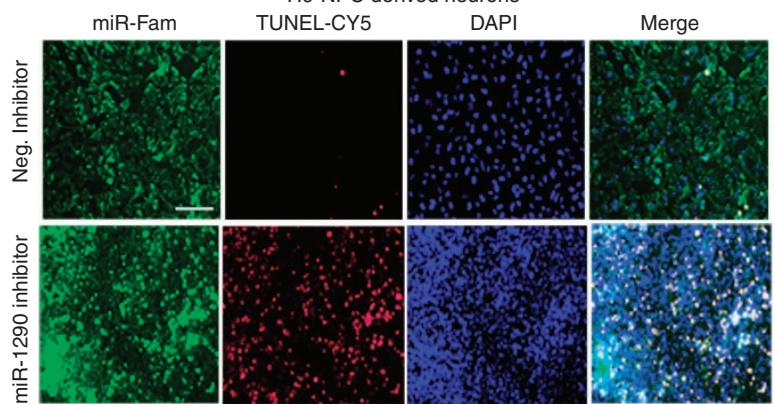

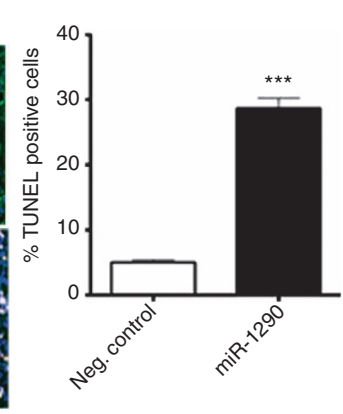

e

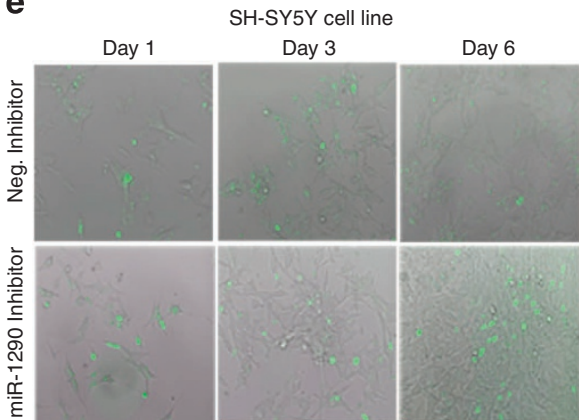

Figure 4 MiR-1290 is required for neuronal differentiation. ( $\mathbf{a}$ and $\mathbf{b}$ ) The miR inhibitor (for miR-1290 and control) in green (miR-FAM)-transfected H9-NPCs were placed in differentiation medium for 5 days and cells were then labeled with BrdU (red, a) and Ki67 (red, b), bar $=20 \mu \mathrm{m}$. Right panels represent increase in \% of BrdU and Ki67 labeling in miR-1290-inhibited cells $(n=3)$. (c) DAPI staining on cells in the presence/absence of miR-1290 and negative control inhibitors showed a drastic increase in the density of cells $(\mathbf{c}$, bar $=20 \mu \mathrm{m})$. Right panel represents increase in the number of DAPI-labeled nuclei in miR-1290-inhibited cells $(n=3)$. (d) TUNEL staining on inhibitor-transfected cells at day 7 in culture, $\mathrm{bar}=20 \mu \mathrm{m}$. Right panel represents $\%$ of mean TUNEL-positive cells $(n=3)$. ${ }^{\star \star} P<0.01,{ }^{\star \star \star} P<0.001$ determined by an unpaired Student's $t$-test. (e) SH-SY5Y cells were differentiated with $10 \mu \mathrm{M}$ RA followed by $10 \mathrm{ng} / \mathrm{ml} \mathrm{BDNF}$ in the presence of the miR inhibitor and monitored during days 1,3 and 6 of differentiation. Note the increase in density of culture and lack of neurite formations in miR-1290 inhibitor-transfected cells

Figure 3 MiR-1290 is upregulated during differentiation. (a) Differentiated hNPC-derived neurons (hNPC-Ns) from neurosphere stained with differentiation marker Tuj-1 (green, top panels) and double labeled with miR-1290 (pink, bottom panel), bar $=20 \mu \mathrm{m}$. (b) Bar graph on the left represents fold increase in miR-1290 during different days of h-NPC differentiation to neurons. Bar graph on the right represents fold increase in miR-1290 expression in differentiated SH-SY5Y cells. (c) H9-NPC-derived neurons were stained with neuronal markers, MAP2, NeuN and Tuj-1 (left panel, bar $=50 \mu \mathrm{m}$ ). Lower-most panels represent ISH images (bar $=20 \mu \mathrm{m}$ ) of H9-NPC-derived neurons during differentiation; particularly, day 7 neurons show localization of miR-1290, not only in cell body and nucleus but also extending into the processes. Bar graph represents fold increase in miR-1290 in H9-NPC differentiated cells (threefold, $P<0.001, n=4$, unpaired Student's $t$-test). ${ }^{\star} P<0.05$, ${ }^{\star \star} P<0.01,{ }^{* \star \star} P<0.001$ determined by one-way ANOVA for (b) 
a
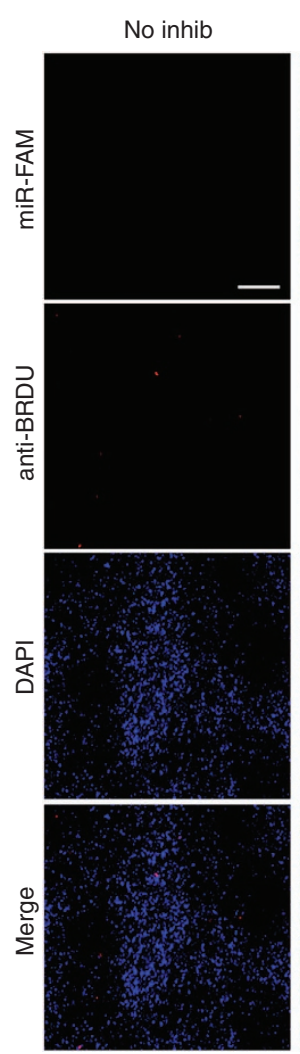

Human primary neurons

Neg Inhibitor
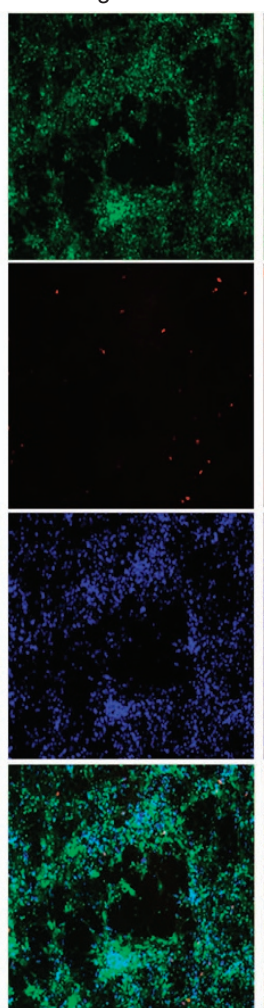

miR-1290 inhibitor
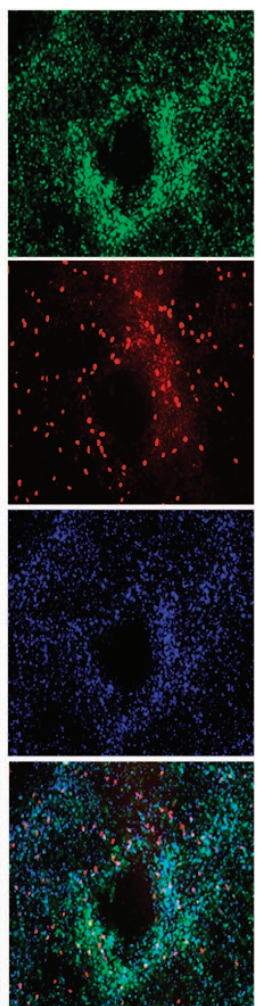

b

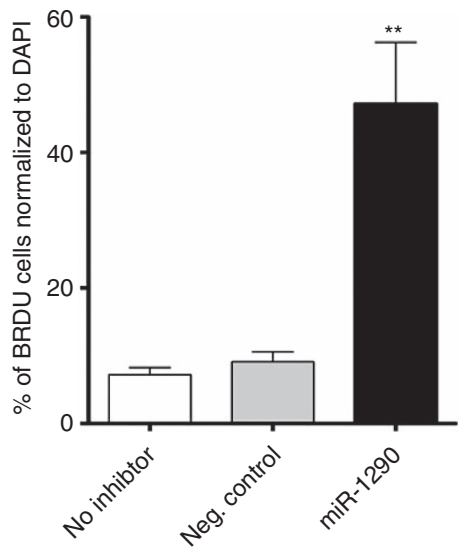

Figure 5 MiR-1290 is required to maintain differentiated hNPC-derived neurons. (a) Neurons were grown in the presence of the miR inhibitor in green (miR-FAM) for 5 days and cells were labeled with $\mathrm{BrdU}$ (red), bar $=20 \mu \mathrm{m}$. (b) Bar graph represents increase in $\%$ of BrdU labeling in miR-1290-inhibited neuronal culture $(n=3)$. ${ }^{* *} P<0.01$ determined by one-way ANOVA

Studies have shown that perturbations in evolutionarily conserved miRNAs can affect crucial aspects of neurogenesis. For example, miR-9 is essential for proliferation and migration of H9-hESC-derived NPCs, ${ }^{19}$ and in mouse NPCs, miR-34a overexpression reduces neuron yield upon differentiation. ${ }^{20}$ In addition, the combination of three miRNAs (miR-9*/miR-9 and miR-124) has been shown to induce conversion of human fibroblasts into neurons. ${ }^{8}$ However, although higher-species specific miRNAs have been found in the brain, ${ }^{10,11,21}$ their functional relevance has been addressed only in few studies. For example, the recently discovered human-specific miR-941 has been identified to play a critical role in pluripotent stem cell proliferation and in neurotransmitter release; ${ }^{21}$ our microarray analysis did not show a significance change in its expression during differentiation. Another young miRNA, miR-1246 (following miR-1290, had next highest significant change in our microarray analysis, upregulated $\sim 10$-fold during differentiation), can target DYRK1A, a Down syndrome-associated protein kinase, and inhibit its activity. ${ }^{22}$ It is interesting to speculate that these higher miRNAs have specific roles in brain functions, and the subtle differences in miRNA sequences are crucial in determining their evolutionary conservation across species and thus might help us to understand their specific functional properties.

One of our models, primary cultures of fetal neurons, do not represent adult mature neurons as seen in adult human brain but can represent neurodevelopmental events taking place in the developing brain. When stained for neuronal markers, the human fetal-derived primary cultures not only expressed MAP2 and NeuN but also markers for immature neurons, Tuj1 and Tuc-4. Interestingly, when we compared ISH signals between adult human brain and fetal brain, miR-1290 signal in neurons is predominantly seen in cytoplasm and nucleus, respectively (Figure 2). This nuclear localization of signal is also seen in neurons differentiated from primary cultures (Figure 3a). Studies on the neuronal miR-124 also reveal both cytoplasmic and nuclear localization. ${ }^{23}$ Evidence suggests that processing of the inactive precursor miRNA into the mature, active form by Dicer may be, in part, regulated by the sequestration of the precursor form, typically in the nucleus. ${ }^{24,25}$ Therefore, it is likely that nuclear signal in immature neurons represents the unprocessed miR-1290 and becomes more cytoplasmic as neuron matures that is evidenced in the adult brain sections. Indeed, this is true as when we analyzed mature miR-1290 levels by qRT-PCR, we saw a gradual increase during days of differentiation in vitro (Figure 3a). Furthermore, northern blot revealed presence of mature miR-1290 in only differentiated cultures. As inhibition of mir-1290 levels led to increased proliferation of cells and overexpression of miR-1290 slowed down cell cycle, we inferred that it has a role in regulating cell cycle. In support of this, miR-1290 was upregulated in senescent human lung fibroblasts when compared with proliferating fibroblasts. ${ }^{26}$ 
The cyclin kinase inhibitor p27 is a central regulator for cell cycle and is vital for neuronal differentiation. Overexpression of p27 arrests cells in $\mathrm{G}_{1},{ }^{27}$ whereas its loss leads to an increase in cell proliferation. ${ }^{28-30}$ Cyclin-dependent kinase 5 (CDK5) directly phosphorylates $\mathrm{p} 27,{ }^{31}$ and is strongly activated in postmitotic neurons. ${ }^{32}$ Loss of CDK5 in mature neurons facilitated the reentry of the neurons into cell cycle. $^{33}$ During neuronal differentiation and migration, a positive feedback loop exists between CDK5, its activator p35 and p27. ${ }^{34}$ Therefore, we speculated changes in these core cell cycle proteins during inhibition or overexpression of miR-1290 in cells. Indeed, we saw a significant upregulation of p27, CDK5 and p35 with miR-1290 inhibition and their downregulation with miR-1290 in levels, suggesting that the functional changes are indeed a result of changes in these proteins. Whether miR-1290 binds directly to or has an indirect effect on these proteins through targeting other upstream mediators is yet to be ascertained.

Although we identified several cell cycle-related genes as putative targets for miR-1290 (Supplementary Table 2), it is indeed hard to attribute a specific role for miR-1290 in controlling cell cycle as its targets are very much spread out as mapped in the KEGG cell cycle pathway (Supplementary Figure 6). However, it is possible to construct a tenable working hypothesis (Figure 8c). There is a direct target, SKP2 (S-phase kinase-associated protein 2, E3 ubiquitin protein ligase), an E3 ubiquitinase that preferentially ubiquitinates phosphorylated p27 and targets it for degradation, thus controlling p27 levels. ${ }^{35-37}$ Hence, inhibition of SKP2 by miR-1290 binding will lead to increased p27 levels and cell cycle exit. Furthermore, we also identified cyclin genes such as cyclin $A$ and $E$ in the cell cycle pathway that are direct targets of miR-1290 and can therefore have an indirect effect on p27, CDK5 and p35 levels.

Reactivation of cell cycle genes in neurons has been found in neurodegenerative diseases such as Alzheimer's Disease, Parkinson's Disease, Amyotrophic Lateral Sclerosis and Ataxia Telangiectasia, where entry into the cell cycle is implicated in untimely neuronal death. ${ }^{38,39}$ Indeed, these findings highlight the need of cell cycle check in neurons and show its failure to be detrimental in adult brain. The neuronal differentiation models used in this study may recapitulate the differentiation of immature neurons as seen in the developing brain; interestingly, reentry into the cell cycle in immature neurons may lead to a quicker onset of apoptotic death than it does in mature neurons. ${ }^{38}$ Intriguingly, delay in cell cycle exit during brain development may underlie the larger brain sizes and increased number of neurons in autism spectrum disorders. ${ }^{40}$ It would be interesting to study the role of miR-1290 in such disorders.

Our study strongly suggests that miR-1290 can play a role in mitotic exit during neuronal differentiation by regulating key cell cycle proteins. Most miRNA studies utilize evolutionarily conserved molecules that readily enable studies in vivo in experimentally tractable animals such as rodents. However, some physiological systems, in particular the brain, have evolved to quite advanced functions in humans compared with rodents. The greatly increased cognitive ability of humans is linked to the enlargement of the frontal cortex, and humans and the other great apes share this disproportionate enlargement. ${ }^{41}$ Determining the unique aspects of brain and neuronal development and differentiation in higher organisms will be key to uncovering the processes leading to these structural and functional differences. The combined and compelling evidence drawn from various model systems further strengthens our study. These exciting studies emphasize the need to study human-specific miRNAs that have specific and central roles in crucial neuronal developmental process and much more subtler processes such as the cell cycle.

In summary, miR-1290 plays a crucial role in neuronal differentiation. Knockdown of mir-1290 increased proliferation and inhibited differentiation, whereas overexpression slowed down the cell cycle and initiated differentiation. The strictly regulated expression patterns of miR-1290 during proliferation and differentiation stages, its expression exclusive to higher species and its interaction with key cell cycle proteins offer strong support for future studies further defining its role in the neuronal fate specification.

\section{Materials and Methods}

Cell culture. Human aborted fetal brain tissue (12-16 weeks post conception), obtained from University of Washington, was handled in full compliance with the University of Nebraska Medical Center (UNMC) and NIH ethical guidelines under IRB approval. Derivation and culturing of NPCs and derived neurons were carried out as described previously. ${ }^{42}$ Briefly, cortex was enzymatically $(0.25 \%$ trypsin) dissociated into a single-cell suspension. The cells were maintained in Ex-Vivo15 (Biowhittaker-Lonza Walkersville Inc., Walkersville, MD, USA) medium supplemented with N2 supplement (Life Technologies, Carlsbad, CA, USA), bFGF $20 \mathrm{ng} / \mathrm{ml}$ (Sigma, St. Louis, MO, USA), EGF $20 \mathrm{ng} / \mathrm{ml}$ (Sigma), LIF $10 \mathrm{ng} / \mathrm{ml}$ (Sigma), NSF-1 (Biowhittaker-Lonza Walkersville Inc.) and $60 \mathrm{ng} / \mathrm{ml} \mathrm{N}$-acetylcarnitine (Sigma). After 3-5 days, cells form clusters/neurospheres of hNPCs, and are further maintained in the above medium until differentiation.

After 7-10 days in culture, hNPCs were differentiated into neurons or astrocytes, which was achieved by gentle mechanical dissociation, if needed; Stempro Accutase (Life Technologies) was used for dissociation. For neurons, dissociated cells were plated onto poly-D-lysine-coated plates in Neurobasal media supplemented with B27 (Life Technologies), $0.5 \mathrm{mM}$ L-glutamine, $10 \mathrm{ng} / \mathrm{ml}$ BDNF (Peprotech Inc., Rocky Hill, NJ, USA) and $10 \mathrm{ng} / \mathrm{ml}$ NT3 (Peprotech). Neurons were grown until DIV 14-21; media was half exchanged as needed. For astrocyte conversion, hNPCs were plated in astrocyte growth media (DMEM, 10\% FBS) for 1 week.

SH-SY5Y cells were differentiated to neuronal-like cells by replacing the media to growth media containing $10 \mu \mathrm{M}$ all-trans retinoic acid (Sigma) for 3 days, followed by 3 days in $50 \mathrm{ng} / \mathrm{ml} \mathrm{BDNF}$.

H9-NPCs were obtained from ArunA Biomedical Inc (Athens, GA, USA) and passaged three times before using them for experiments. H9-NPCs were cultured on Matrigel (BD Biosciences, Sparks, MD, USA) in the presence of growth media (ArunA Biomedical) supplemented with $20 \mathrm{ng} / \mathrm{ml} \mathrm{bFGF}$. For differentiation, cells were plated on Matrigel and bFGF was not added to the growth media; instead, we added $20 \mathrm{ng} / \mathrm{ml} \mathrm{NT3}$ and $20 \mathrm{ng} / \mathrm{ml}$ BDNF to sustain the differentiated neurons. Differentiation was typically carried for 7 DIV.

Antibodies and immunofluorescence. Immunofluorescence was performed on $4 \%$ paraformaldehyde (PFA)-fixed cells permeabilized with $0.25 \%$ Tween-20. Primary antibodies were mouse anti-Nestin (Millipore, Billerica, MA, USA), rabbit anti-Sox2 (Millipore), mouse anti-MAP2 (Covance, Princeton, NJ, USA), rabbit anti-GFAP (DAKO, Carpinteria, CA, USA), rabbit anti-Mushashi1 (Millipore), rabbit anti-Tuj1 (Millipore), mouse anti-NeuN (Millipore), mouse antiOct4 (Millipore), rabbit anti-Pax6 (Covance), rabbit anti-TUC4 (Millipore), mouse anti-Vglut1 (Synaptic Systems, Goettingen, Germany), mouse anti-BrdU (SBCT, Santa Cruz, CA, USA) and rabbit anti-Ki67 (Abcam, Cambridge, MA, USA). Species-specific Alexa-Fluor-labeled secondary antibodies was used for detection followed by mounting in Prolong gold anti-fade with DAPI (Life Technologies). 
a
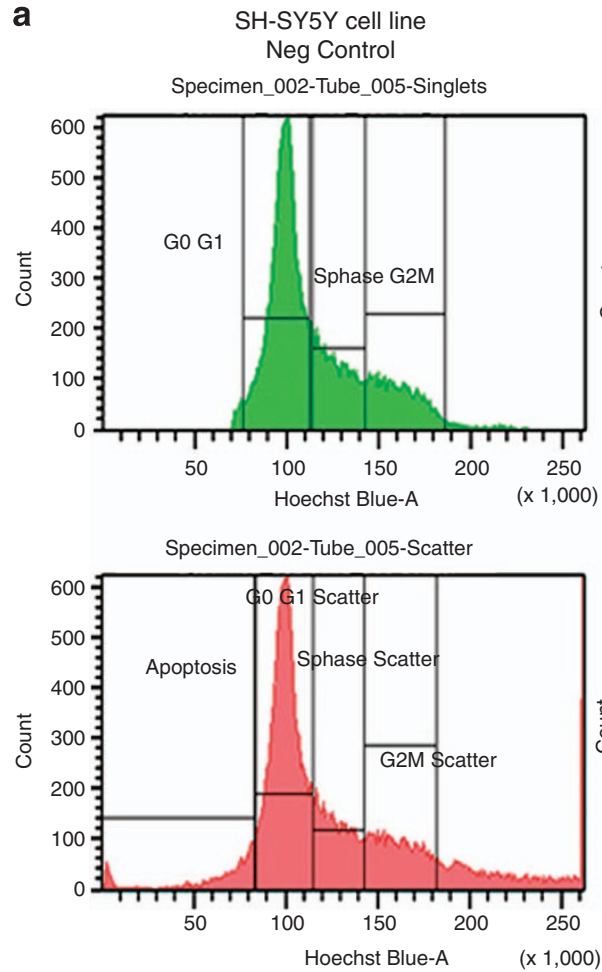

$\operatorname{miR}-1290$
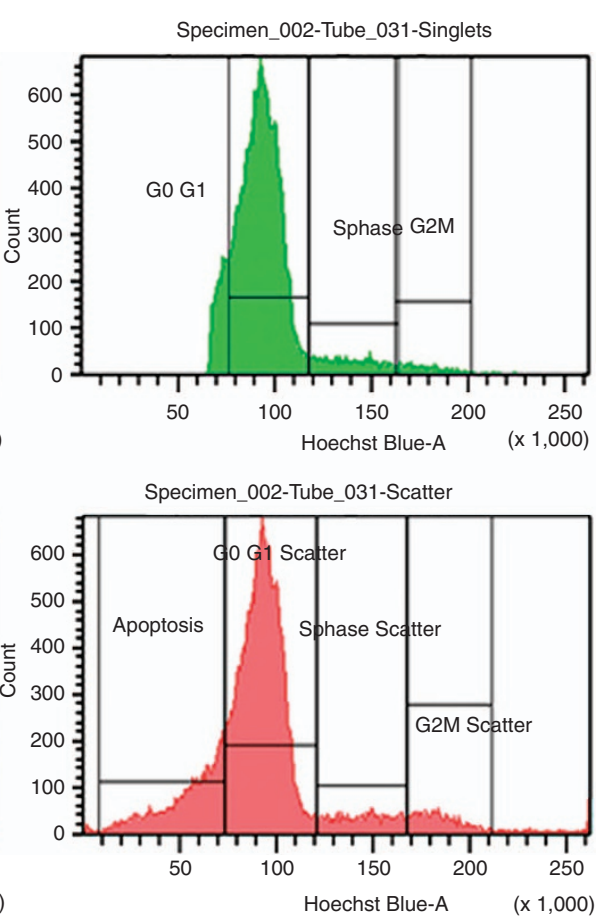
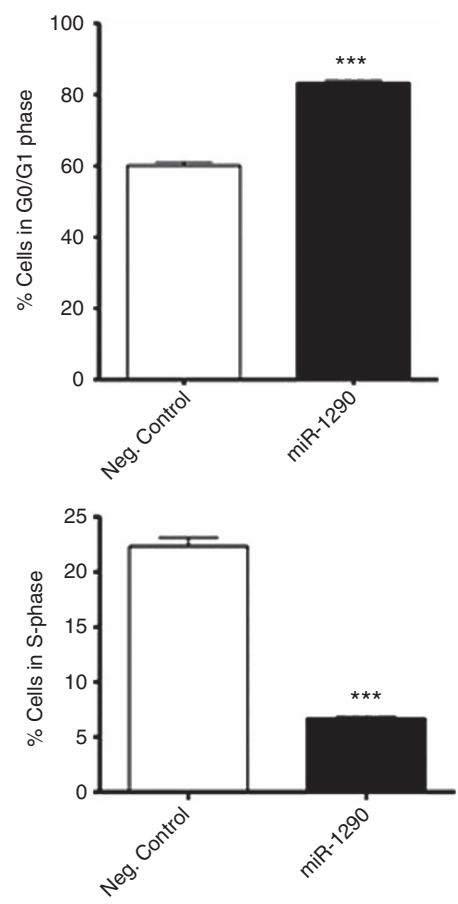

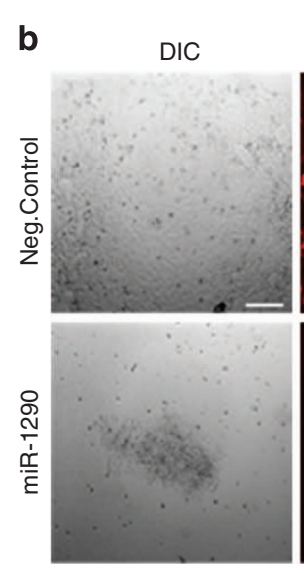

C

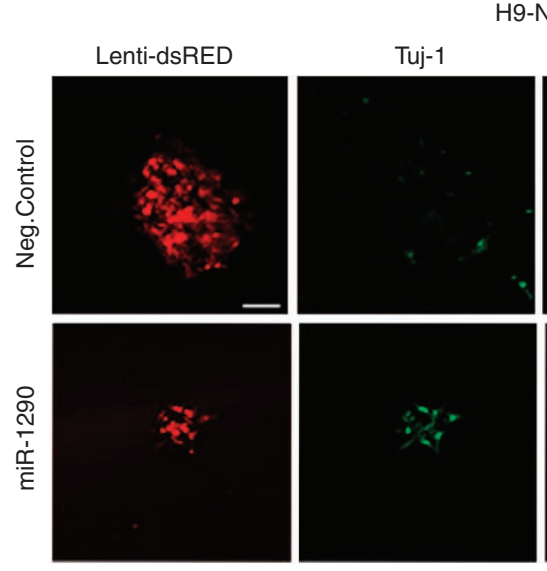

9-NPC

H9-NPC

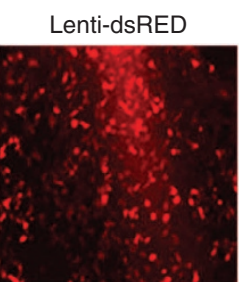

Merge
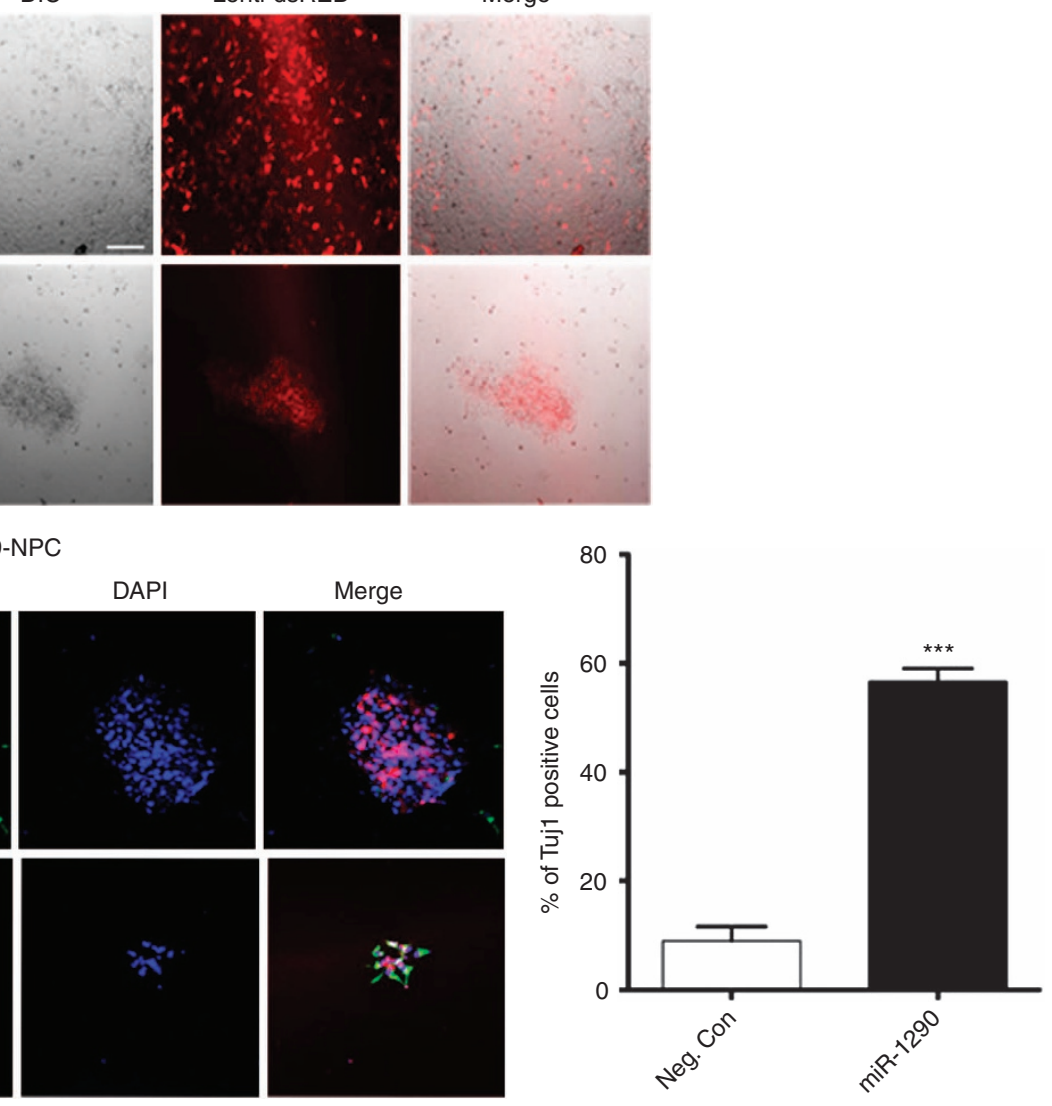
Proliferation analysis by BrdU staining. BrdU staining was performed as described before. ${ }^{43}$ Briefly, BrdU $(10 \mu \mathrm{M})$ was added to the cells and incubated for $4 \mathrm{~h}$ at $37^{\circ} \mathrm{C}$ and fixed with $4 \%$ PFA for $15 \mathrm{~min}$. Next, the fixed cells were incubated in $2 \mathrm{~N} \mathrm{HCl}$ with $0.3 \%$ Triton $\mathrm{X}-100$ for 30 min followed by $0.1 \mathrm{M}$ boric acid (pH 8.0) for $10 \mathrm{~min}$ and three rinses with PBS before incubation blocking solution $(0.3 \%$ Triton $X-100,1 \%$ bovine serum albumin and $3 \%$ normal goat serum in PBS) for $1 \mathrm{~h}$. Next, the cells were incubated with a mouse monoclonal anti-BrdU antibody $(1: 100)$ in the blocking solution overnight at $4^{\circ} \mathrm{C}$. After three PBS rinses, the Alexa Fluor 568 goat anti-mouse secondary antibody $(1: 500)$ was applied in blocking solution for $4 \mathrm{~h}$ at room temperature. The cells were rinsed in PBS three times and visualized using a fluorescent microscope and images were taken. Total number of BrdU-labeled cells was calculated by normalizing the BrdU signals to the nuclei stain, DAPI. ImageJ software (http://rsb.info.nih.gov/ij/index.html) was used for counting the cells in a given field.

RNA isolation and microarray. RNA was isolated using Trizol (Life Technologies). The miRNA microarray was performed on Affymetrix GeneChip miRNA 2.0 Array. GeneChip miRNA arrays were run by the UNMC microarray core using the manufacturer's recommended equipment and protocols. Data were analyzed using Partek genomics suite (Partek Inc., St. Louis, MO, USA).

Quantitative real-time PCR. TaqMan mature miR assays for hsa-miR-1290 (Applied Biosystems, Carlsbad, CA, USA) was used to quantify according to the manufacturer's protocol. U6 snRNA was used as housekeeping control. Reactions were performed and calculations made as described using the $2^{-\Delta \Delta C T}$ method. ${ }^{44}$

miRNA ISH. Brain tissue sections were obtained for normal adult and fetal brains from BioChain (BioChain Institute, Inc., Hayward, CA, USA). Tissue sections were deparaffinized and hydrated before citrate treatment $(10 \mathrm{mM}$ citrate, $\mathrm{pH} \mathrm{6.39)}$ at $90^{\circ} \mathrm{C}$ for $40 \mathrm{~min}$ followed by washes in $1 \times$ PBS. Next, sections were washed in freshly prepared methlyimidazole buffer containing $130 \mathrm{mM}$ 1-methlyimidazole, $300 \mathrm{mM} \mathrm{NaCl}, \mathrm{pH} 8.0$, followed by 1 -h incubation with $160 \mathrm{mM}$ EDC (1-ethly-3-(3-dimethlyaminopropyl)) pH 8.0 solution. Tissue culture cells on coverslips were fixed in $4 \%$ PFA, coverslips were incubated overnight with $70 \%$ ethanol and permeabilized using $0.25 \%$ Triton-X-100 for 15 min followed by two washes in $2 \times$ SSC. All specimens were then prehybridized in hybridization buffer (50\% formamide, $10 \mathrm{mM}$ Tris-HCl, pH 8.0, $200 \mu \mathrm{g} / \mathrm{ml}$ yeast tRNA, $1 \times$ Denhardt's solution, $600 \mathrm{mM} \mathrm{NaCl}, 0.25 \%$ SDS, $1 \mathrm{mM}$ EDTA and $10 \%$ dextran sulfate) for $1 \mathrm{~h}$ at $37^{\circ} \mathrm{C}$ in a humidified chamber. LNA-modified miR-1290, U6 snRNA and scrambled miR probes, labeled at both the $5^{\prime}$ and $3^{\prime}$ ends with digoxigenin (Exigon, Vedbaek, Denmark), were diluted to a final concentration of $4 \mathrm{pM}$, heated to $65^{\circ} \mathrm{C}$ for $5 \mathrm{~min}$ and separately hybridized at $37^{\circ} \mathrm{C}$ overnight. Specimens were then washed twice in hybridization buffer (without probe) at $37^{\circ} \mathrm{C}$, followed by washing three times in $2 \times$ SSC and twice in $0.2 \times$ SSC at $42^{\circ} \mathrm{C}$. They were then blocked with $1 \%$ BSA, $3 \%$ normal goat serum in $1 \times$ PBS for $1 \mathrm{~h}$ at room temperature and incubated with anti-digoxigenin conjugated with horseradish peroxidase (Roche Applied Science, Indianapolis, IN, USA). The following day, sections were washed with two PBS washes and signal amplification (for the in situ, now labeled with horseradish peroxidase) using TSA Cy5 kit (PerkinElmer, Waltham, MA, USA) according to the manufacturer's protocol. The slides were mounted in Prolong gold anti-fade reagent with DAPI (Life Technologies).

Double label in situ/immunofluorescence was performed as previously described. ${ }^{44,45}$ In order to examine the potential for false-positive signals arising because of nonspecific probe hybridization to the nuclear DNA, sections were treated with RNAse A (100 $\mu \mathrm{g} / \mathrm{ml})$ for $1 \mathrm{~h}$ at $37^{\circ} \mathrm{C}$ before the hybridization step.

miRNA northern blotting. Northern blotting for miRNA detection was performed with minor modifications as described previously. ${ }^{46,47}$ Briefly, $10 \mu \mathrm{g}$ of RNA denatured in Ambion gel loading buffer II (Life technologies) at $75^{\circ} \mathrm{C}$ for $15 \mathrm{~min}$ was loaded and ran on a 15\% TBE-Urea gels (Invitrogen, Carlsbad, CA, USA) at
$180 \mathrm{~V}$ for $1 \mathrm{~h}$. The gel was then transferred onto a nylon membrane using an iBLOT DNA transfer stack (Invitrogen) as per the manufacturer's instructions. Subsequently, the membrane was crosslinked at $1200 \mathrm{~kJ}$ using a STRATALINKER (Stratagene, La Jolla, CA, USA). The membrane was then prehybridized in the prehyb buffer (Sigma) at $37^{\circ} \mathrm{C}$ for $1 \mathrm{~h}$ followed by addition of $1.2 \mathrm{pmol} / \mathrm{ml}$ of LNA-modified $5^{\prime}$ and $3^{\prime}$ DIG-labeled hsa-miR-1290 probe (Exiqon) to the buffer and hybridization overnight at $37^{\circ} \mathrm{C}$. The next day, the membrane was washed for 5 min with a low stringency wash buffer $(2 \times$ SSC, $0.1 \%$ SDS) followed by $2 \times$ washes for 20 min each with a high stringency wash buffer $(0.5 \times \mathrm{SSC}, 0.1 \% \mathrm{SDS})$ and a final wash for $20 \mathrm{~min}$ with ultrahigh stringency wash buffer $(0.1 \times \mathrm{SSC}, 0.1 \% \mathrm{SDS})$. After the washes, the membrane was blocked for $1 \mathrm{~h}$ with $1 \times$ blocking buffer for $1 \mathrm{~h}$ followed by incubation with Anti-DIG AP antibody in a 1:20000 dilution in blocking buffer. Finally, DIG signal development was carried out using the DIG wash and block buffer set.

Knockdown and overexpression of miR-1290. The LNA-based miRNA power inhibitors (with or without $5^{\prime}$-FAM label) were purchased from Exiqon. Undifferentiated SH-SY5Y cells and H9-NPCs were plated on coverslips or in six-well plates, at $70 \%$ confluency, and $25 \mathrm{nM}$ of the unlabeled/FAM-labeled miR-1290 or the scrambled inhibitor (Negative control) was transfected using XtremeGENE-siRNA (Roche Applied Science) as per the manufacturer's instructions. The cells were differentiated in the presence of the inhibitor. At the end of 6 days (for SH-SY5Y) and 5 days (H9-NPCs), coverslips were placed in 4\% PFA for staining and cells on plates were harvested in Trizol for RNA extraction. Cells transfected with FAM-labeled probes were monitored daily for phenotypic changes. For knockdown experiments on human neurons derived from primary fetal cells (hNPC-Ns), neurons were grown in culture for 21 days until fully differentiated and then transfected with inhibitor as described above. Coverslips were fixed in 4\% PFA at the end of 5 days for analysis.

For overexpression studies, the undifferentiated SH-SY5Ys or H9-NPCs were transduced with lentivirus expressing miR-1290 (Biosettia, San Diego, CA, USA) and were maintained in proliferative growth media.

Western blotting. Whole-cell lysates were prepared using RIPA buffer $(50 \mathrm{mM}$ Tris $/ \mathrm{HCl}, \mathrm{pH} 8 ; 150 \mathrm{mM} \mathrm{NaCl} ; 1 \%$ Nonidet P-40; $0.5 \%$ sodium deoxycholate; and $0.1 \%$ SDS) and protein quantification was carried out using Pierce BCA protein assay (Thermo Scientific, Rockford, IL, USA). Protein (5$15 \mu \mathrm{g}$ ) was loaded in each lane of NuPAGE 4-12\% Bis-Tris gels (Invitrogen). Separated proteins were transferred onto nitrocellulose membranes using iBlot (Invitrogen). The membranes were blocked in SuperBlock (TBS) blocking buffer (Thermo Scientific) and then incubated overnight at $4^{\circ} \mathrm{C}$ with primary antibody. The following primary antibodies were used: rabbit polyclonal p27kip1 $(1: 500$, Abcam), rabbit polyclonal p35 $(1: 500, \mathrm{SBCT})$, mouse monoclonal CDK5 $(1: 1000$, Millipore), mouse monoclonal PCNA (1:1000, DAKO) and rabbit polyclonal actin (1:5000; Sigma-Aldrich, St. Louis, MO, USA). This was followed by incubation with secondary antibody, HRP conjugated anti-rabbit IgG $(1: 20000$; Thermo Scientific) and anti-mouse IgG (1:20000; Thermo Scientific) for $1 \mathrm{~h}$ at room temperature. Blots were developed using SuperSignal West Pico Chemiluminescent Substrate (Thermo Scientific), imaged and quantified using Carestream MI software (Carestream Health INC, Rochester, NY, USA).

Preparation of miR-1290-expressing lentivirus. The miR-1290expressing lentiviral vector (pLV-miR-1290) and the packaging mix (LTR-pack-1 and LTR-pack-2) were purchased from Biosettia and viral preparations were done as per the instructions. Briefly, human embryonic kidney (HEK) 293T cells were transfected with pLV-miR1290, LTR-pack-1 and LTR-pack-2 using XtremeGene HP transfection reagent (Roche Applied Science) according to the manufacturer's protocol. Cell supernatant containing virions was collect 48 and $72 \mathrm{~h}$ after transfection, and concentrated by ultracentrifugation. pLV vector without insert was used as control. Lentivirus titer was determined using HIV p24 ELISA assay (Express Biotech International, Thurmont, MD, USA).

Figure 6 MiR-1290 overexpression leads to slowdown of cell cycle in SH-SY5Y cells, reduces the colony formation and induces differentiation in H9-NPCs. (a) Flow cytometry histograms on the right reveal that SH-SY5Y cells transduced with a lentivirus construct expressing miR-1290 show an increased proportion in G0/G1 phase and a lower proportion in the S phase (bottom), representing the slowing down of cell proliferation $(n=3)$. (b) H9-NPCs were transduced with a lentivirus construct expressing miR-1290 or a negative control sequence. Transduced cells selected with puromycin show a drastic decrease in colony size and cell number in miR-1290-transduced cells when compared with negative (Neg.) control, bar $=50 \mu \mathrm{m}$. (c) Immunostaining the colonies with differentiation marker, Tuj-1, showed increased staining in miR-1290-transduced cells, bar $=20 \mu \mathrm{m}$. Bar graph represents increase in \% of Tuj1-positive labeling in miR-1290-overexpressed H9-NPCs $(n=3)$. ${ }^{* \star} P<0.001$ determined by unpaired Student's t-test 
SH-SY5Y cells or H9-NPCs were grown in vitro till they achieve $80 \%$ confluency and transduced with miR-1290 or control lentivirus at a concentration of $5 \times 10^{6}$ lentiviral particles/ml. Successful transduction was confirmed by visualizing dsRED expression. At 5 days after transduction, cells were visualized in Zeiss Observer.Z1 microscope equipped with Axiocam MRm camera using Axiovision 40 v.4.8.0.0 software (CarlZeiss, Oberkochen, Germany).

a
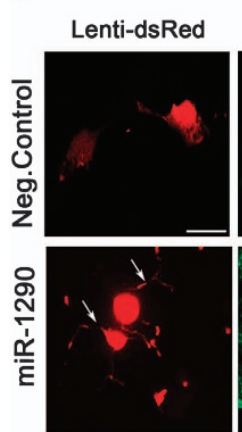

b Lenti-dsRed

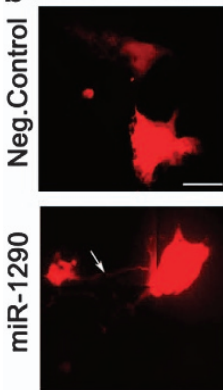

C Lenti-dsRed
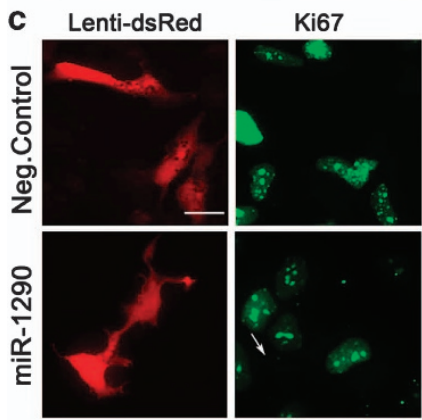

DAP
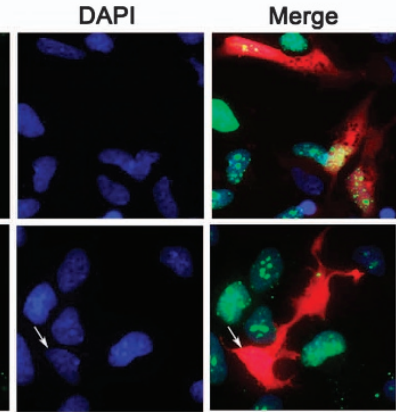

d

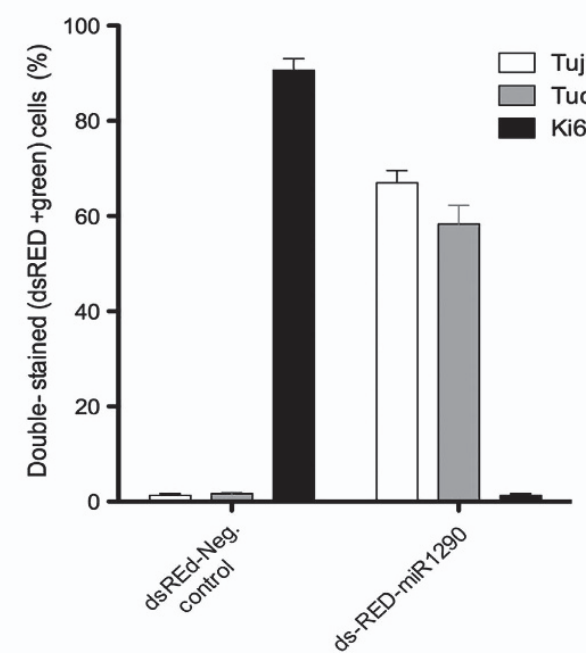

Proliferation assay. Numeric grid-marked coverslips (Bellco Biotechnology, Vineland, NJ, USA) coated with Matrigel were used to track proliferation. The numbers on the grid were used to identify the grid squares that had equivalent cell densities before transfections. In order to verify the proliferation of H9-NPCs, cells were plated at a low density (20000 cells/coverslip) and transfected with FAMlabeled miR-1290 or negative control inhibitors (Exiqon). Cells were differentiated for 5 days post transfection and fixed in 4\% PFA. Each square with equivalent number of cells was imaged for nuclei stain DAPI. Images were then exported to ImageJ software for further analysis. The number of DAPI-stained nuclei from negative control inhibitor and Mir-1290 inhibitor-transfected squares $(n=4)$ were normalized to no inhibitor control and the values were represented as percentages in the bar graphs.

Bioinformatics. The mRNA transcripts were extracted from ENSEMBL. The miR-1290 gene targets were filtered by mir-1290 seed region ( $5^{\prime}$-AAAUCCA- $\left.3^{\prime}\right)$ complementarity in the $3^{\prime}$-UTR. To identify the biological networks or pathways, the FatiGO tool in the Babelomics suite (http://babelomics.bioinfo.cipf.es/) was utilized. ${ }^{48}$ FatiGO uses Fisher's exact test to check for significant overrepresented GO terms in a group of genes.

Transfection of fosmid DNA. NIH3T3 cells were grown in glass coverslips or six-well plates and transfected with fosmid clone (G248P89639D9, http:// bacpac.chori.org) by Fugene-HD (Roche Applied Sciences) as per the manufacturer's instructions. After $48 \mathrm{~h}$ of transfection, coverslips were fixed with $4 \%$ PFA and wells harvested for RNA.

Flow cytometry for cell cycle analysis. Cells were stained with Hoechst stain, assessed by flow cytometry and cell cycle was measured as described previously. ${ }^{49}$

TUNEL staining. TUNEL staining was performed as described previously. ${ }^{50}$ Briefly, ApopTag Plus Peroxidase In situ Apoptosis Detection kit (Millipore) was used according to the manufacturer's instructions with minor modifications. Cells were fixed on coverslips with $1 \%$ paraformaldehyde, followed by washes and postfixation for $5 \mathrm{~min}$ at $-20^{\circ} \mathrm{C}$ with ethanol/acetic acid (2:1), followed by PBS washes. Samples were reacted for 5 min with $3 \% \mathrm{H}_{2} \mathrm{O}_{2}$ in PBS to deplete endogenous peroxidase activity and then washed with PBS. Slides were next prehybridized in equilibration buffer for $5 \mathrm{~min}$, followed by hybridization with terminal deoxynucleotidyl transferase enzyme for $1 \mathrm{~h}$ at $37^{\circ} \mathrm{C}$. The hybridization reaction was terminated by incubation with stop buffer and PBS wash. HRPconjugated anti-digoxigenin was added next ( $30 \mathrm{~min}$ at RT), followed by PBS wash. The signal was converted to fluorescence using TSA Cy5 kit (PerkinElmer) according to the manufacturer's protocol. Coverslips were mounted in Prolong gold anti-fade reagent with DAPI (Invitrogen).

Statistical analysis. Statistical analysis was performed using GraphPad Prism software (La Jolla, CA, USA). Student's t-test or one-way ANOVA followed by Dunnett's multiple comparison tests were performed and the $P$-value was calculated for each experiment. For all experiments with error bars, S.D. was calculated to indicate the variation between experiments.

Data deposition accession numbers: The microarray data have been deposited in NCBl's gene expression omnibus (GEO) accession number GSE34016 (http://www.ncbi.nlm.nih.gov/geo/query/acc.cgi).

Figure 7 MiR-1290 overexpression in H9-NPCs leads to the expression of differentiation markers and proliferative arrest. (a and $\mathbf{b}$ ) H9-NPCs were transduced with a lentivirus construct expressing miR-1290 and a negative control sequence. Immunostaining on transduced cells with differentiation marker Tuj-1 and postmitotic marker Tuc 4 shows increased expression in miR-1290 cells, indicating that miR-1290 drives differentiation of proliferating cells, $b a r=10 \mu \mathrm{m}$. (c) Immunostaining on transduced cells with proliferation marker, Ki67, reveals that miR-1290-expressing cells stain negative for Ki67, indicating the loss of proliferative capacity when compared with negative controls (Neg. Control), bar $=10 \mu \mathrm{m}$. (d) The miR-1290-transduced H9-NPCs coexpress the differentiation markers TUC4 and Tuj1, whereas the Neg. control-transduced cells only show coexpression with proliferation marker Ki67. Postimage processing was done for DAPI (Blue) channel in Adobe Photoshop as the signal was weak during capture. Data are represented as $\%$ of total DAPI + cells under each condition 
a
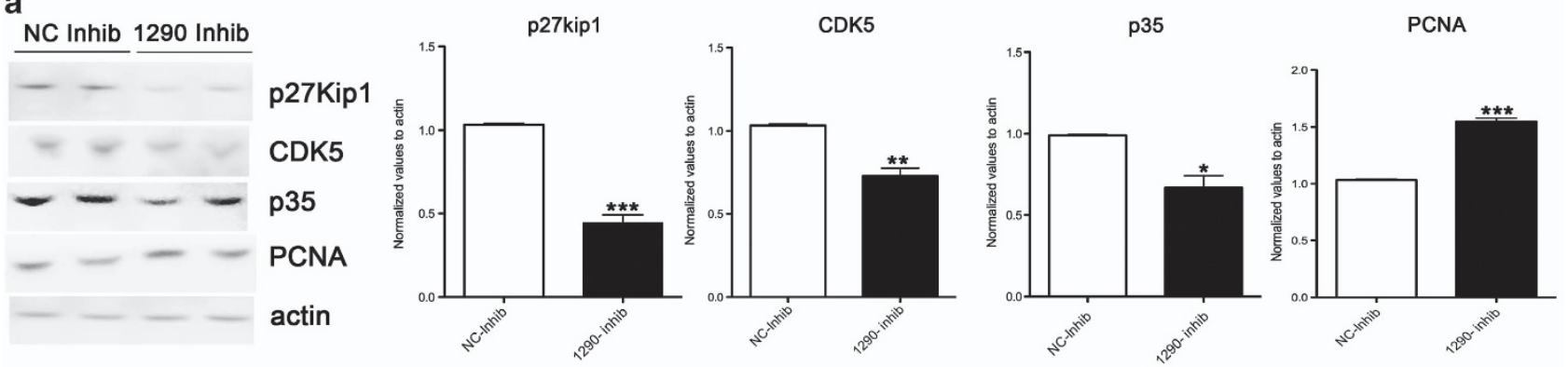

b
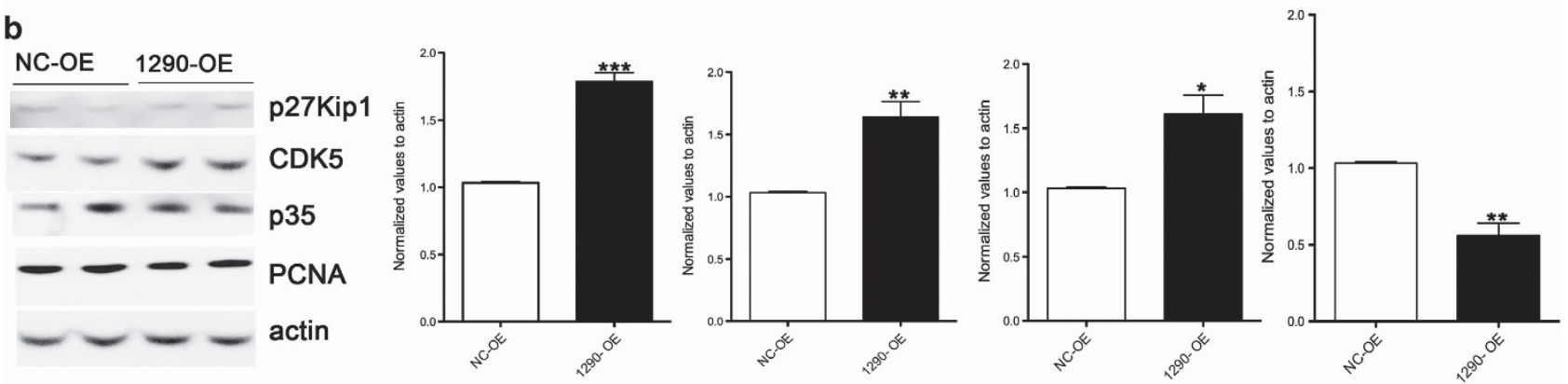

c
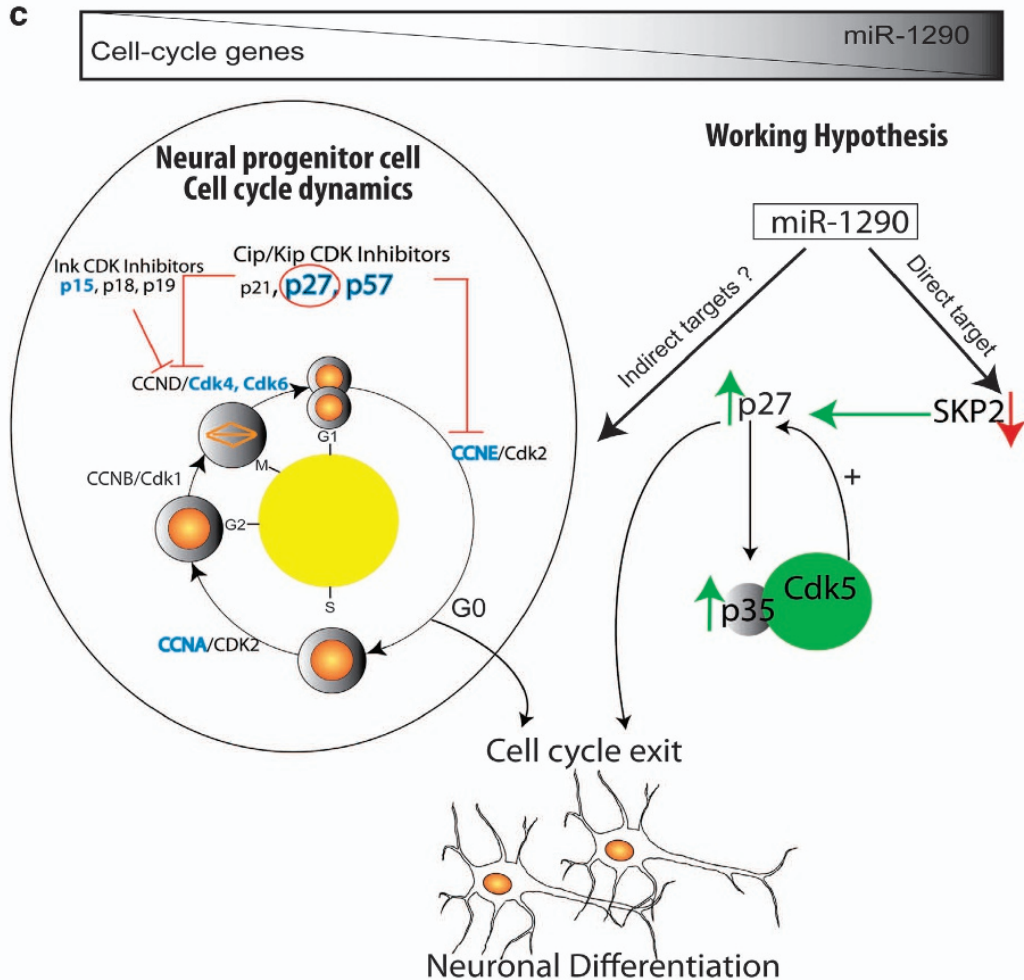

Figure 8 Mir-1290 affects cell cycle proteins. (a) H9-NPCs were differentiated in the presence of miR inhibitors: Neg. Control (NC inhib) or miR-1290 (1290 inhib). Western blots were run on the protein lysates and probed for p27kip1, CDK5, p35 and PCNA; actin was used as an internal control. Note the decrease in the levels of cell cycle proteins in the presence of miR-1290 inhibitor. Bar graphs on the right represent statistical analysis for the protein levels normalized to the internal control, actin. (b) H9-NPCs were transfected with plasmids overexpressing a Neg. Control or miR-1290 sequence for overexpression. Western blots were run on the protein lysates and probed for p27kip1, CDK5, p35 and PCNA; actin was used as an internal control. Note the increase in the levels of cell cycle proteins during miR-1290 overexpression. Bar graphs on the right represent statistical analysis for the protein levels normalized to the internal control, actin. ${ }^{\star} P<0.05,{ }^{\star \star} P<0.01,{ }^{\star \star \star} P<0.001$ determined by unpaired Student's $t$-test. (c) Schematic representation of a neural progenitor cell and its cell cycle dynamics involving core cell cycle regulators, specifically combinations of CDK/cyclin heterodimers allowing the progression of cell cycle. Proteins belonging to the Ink family; p15 Ink-4b, p18 Ink-4c and p19 Ink-4d, and Cip/Kip family: p27kip1, p21cip1 and p57kip2, of CDKs promote cell cycle withdrawal by blocking the activity of cyclins. Proteins in blue represent miR-1290 targets in the core cell cycle regulation. A dual function of miR-1290 is represented in the working hypothesis on the right. MiR-1290 can target and therefore inhibit the expression of SKP2, an E3 ubiquitinase ligase, thereby increasing p27kip1 levels in the cell favoring mitotic cell exit, or can indirectly effect the levels of p27kip1 by binding to cyclin A and cyclin E (CCNA and CCNE) and therefore inhibit cell cycle progression 


\section{Conflict of Interest}

The authors declare no conflict of interest.

Acknowledgements. We thank Benjamin Lamberty and Katy Emanuel for outstanding technical assistance. This work is supported by grants to HSF from the National Institute of Mental Health and Nebraska Tobacco Settlement Biomedical Research Development funds.

\section{Author Contributions}

This study was conceived by SVY and designed by HSF and SVY; SVY performed experiments with BM, DAR, EBH (hNPC and tissue culture), KE (RNA and qRT-PCR), and IT and DRB (bioinformatics); SVY and HSF wrote the paper with comments from all authors.

1. Chua JH, Armugam A, Jeyaseelan K. MicroRNAs: biogenesis, function and applications. Curr Opin Mol Ther 2009; 11: 189-199.

2. Fineberg SK, Kosik KS, Davidson BL. MicroRNAs potentiate neural development. Neuron 2009; 64: 303-309.

3. Kawahara $\mathrm{H}$, Imai $\mathrm{T}$, Okano $\mathrm{H}$. MicroRNAs in neural stem cells and neurogenesis. Front Neurosci 2012; 6: 30.

4. De Pietri Tonelli D, Pulvers JN, Haffner C, Murchison EP, Hannon GJ, Huttner WB. miRNAs are essential for survival and differentiation of newborn neurons but not for expansion of neural progenitors during early neurogenesis in the mouse embryonic neocortex. Development 2008; 135: 3911-3921.

5. Kawase-Koga Y, Otaegi G, Sun T. Different timings of Dicer deletion affect neurogenesis and gliogenesis in the developing mouse central nervous system. Dev Dyn 2009; 238: 2800-2812.

6. Makeyev EV, Zhang J, Carrasco MA, Maniatis T. The MicroRNA miR-124 promotes neuronal differentiation by triggering brain-specific alternative pre-mRNA splicing. Mol Cell 2007; 27: 435-448.

7. Yoo AS, Staahl BT, Chen L, Crabtree GR. MicroRNA-mediated switching of chromatinremodelling complexes in neural development. Nature 2009; 460: 642-646.

8. Yoo AS, Sun AX, Li L, Shcheglovitov A, Portmann T, Li Y et al. MicroRNA-mediated conversion of human fibroblasts to neurons. Nature 2011; 476: 228-231.

9. Rajewsky N. microRNA target predictions in animals. Nat Genet 2006; 38(Suppl): S8-S13.

10. Berezikov E, Thuemmler F, van Laake LW, Kondova I, Bontrop R, Cuppen E et al. Diversity of microRNAs in human and chimpanzee brain. Nat Genet 2006; 38: 1375-1377.

11. Bentwich I, Avniel A, Karov Y, Aharonov R, Gilad S, Barad O et al. Identification of hundreds of conserved and nonconserved human microRNAs. Nat Genet 2005; 37: 766-770.

12. Zheng GX, Ravi A, Gould GM, Burge CB, Sharp PA. Genome-wide impact of a recently expanded microRNA cluster in mouse. Proc Natl Acad Sci USA 2011; 108: 15804-15809.

13. Blanchette M, Kent WJ, Riemer C, Elnitski L, Smit AF, Roskin KM et al. Aligning multiple genomic sequences with the threaded blockset aligner. Genome Res 2004; 14: 708-715.

14. Perelman P, Johnson WE, Roos C, Seuanez HN, Horvath JE, Moreira MA et al. A molecular phylogeny of living primates. PLoS Genet 2011; 7: e1001342.

15. Morin RD, O'Connor MD, Griffith M, Kuchenbauer F, Delaney A, Prabhu AL et al. Application of massively parallel sequencing to microRNA profiling and discovery in human embryonic stem cells. Genome Res 2008; 18: 610-621.

16. Stark MS, Tyagi S, Nancarrow DJ, Boyle GM, Cook AL, Whiteman DC et al. Characterization of the melanoma miRNAome by deep sequencing. PLoS One 2010; 5 : e9685.

17. Witten D, Tibshirani R, Gu SG, Fire A, Lui WO. Ultra-high throughput sequencing-based small RNA discovery and discrete statistical biomarker analysis in a collection of cervical tumours and matched controls. BMC Biol 2010; 8: 58.

18. Chen K, Rajewsky N. The evolution of gene regulation by transcription factors and microRNAs. Nat Rev Genet 2007; 8: 93-103.

19. Delaloy C, Liu L, Lee JA, Su H, Shen F, Yang GY et al. MicroRNA-9 coordinates proliferation and migration of human embryonic stem cell-derived neural progenitors. Cell Stem Cell 2010; 6: 323-335

20. Fineberg SK, Datta $P$, Stein CS, Davidson BL. MiR-34a represses Numbl in murine neural progenitor cells and antagonizes neuronal differentiation. PLoS One 2012; 7: e38562.

21. Hu HY, He L, Fominykh K, Yan Z, Guo S, Zhang X et al. Evolution of the human-specific microRNA miR-941. Nat Commun 2012; 3: 1145.

22. Zhang Y, Liao JM, Zeng SX, Lu H. p53 downregulates Down syndrome-associated DYRK1A through miR-1246. EMBO Rep 2011; 12: 811-817.

23. de Planell-Saguer M, Rodicio MC, Mourelatos Z. Rapid in situ codetection of noncoding RNAs and proteins in cells and formalin-fixed paraffin-embedded tissue sections without protease treatment. Nat Protoc 2010; 5: 1061-1073.

24. Nuovo GJ. In situ detection of precursor and mature microRNAs in paraffin embedded, formalin fixed tissues and cell preparations. Methods 2008; 44: 39-46.
25. Lee EJ, Baek M, Gusev Y, Brackett DJ, Nuovo GJ, Schmittgen TD. Systematic evaluation of microRNA processing patterns in tissues, cell lines, and tumors. RNA 2008; 14: 35-42.

26. Dhahbi JM, Atamna H, Boffelli D, Magis W, Spindler SR, Martin DI. Deep sequencing reveals novel microRNAs and regulation of microRNA expression during cell senescence. PLoS One 2011; 6: e20509.

27. Coats S, Flanagan WM, Nourse J, Roberts JM. Requirement of p27Kip1 for restriction point control of the fibroblast cell cycle. Science 1996; 272: 877-880.

28. Kiyokawa H, Kineman RD, Manova-Todorova KO, Soares VC, Hoffman ES, Ono M et al. Enhanced growth of mice lacking the cyclin-dependent kinase inhibitor function of p27(Kip1). Cell 1996; 85: 721-732.

29. Fero ML, Rivkin M, Tasch M, Porter P, Carow CE, Firpo E et al. A syndrome of multiorgan hyperplasia with features of gigantism, tumorigenesis, and female sterility in p27(Kip1)deficient mice. Cell 1996; 85: 733-744.

30. Nakayama K, Ishida N, Shirane M, Inomata A, Inoue $\mathrm{T}$, Shishido $\mathrm{N}$ et al. Mice lacking p27(Kip1) display increased body size, multiple organ hyperplasia, retinal dysplasia, and pituitary tumors. Cell 1996; 85: 707-720.

31. Kawauchi T, Chihama K, Nabeshima Y, Hoshino M. Cdk5 phosphorylates and stabilizes p27kip1 contributing to actin organization and cortical neuronal migration. Nat Cell Biol 2006; 8: 17-26.

32. Tsai LH, Takahashi T, Caviness VS Jr, Harlow E. Activity and expression pattern of cyclin-dependent kinase 5 in the embryonic mouse nervous system. Development 1993; 119: 1029-1040.

33. Cicero S, Herrup K. Cyclin-dependent kinase 5 is essential for neuronal cell cycle arrest and differentiation. J Neurosci 2005; 25: 9658-9668.

34. Kawauchi T, Hoshino M. Molecular pathways regulating cytoskeletal organization and morphological changes in migrating neurons. Dev Neurosci 2008; 30: 36-46.

35. Carrano AC, Eytan E, Hershko A, Pagano M. SKP2 is required for ubiquitin-mediated degradation of the CDK inhibitor p27. Nat Cell Biol 1999; 1: 193-199.

36. Sutterluty $H$, Chatelain E, Marti A, Wirbelauer C, Senften M, Muller U et al. p45SKP2 promotes p27Kip1 degradation and induces S phase in quiescent cells. Nat Cell Biol 1999; 1: 207-214.

37. Tsvetkov LM, Yeh KH, Lee SJ, Sun H, Zhang H. p27(Kip1) ubiquitination and degradation is regulated by the SCF(Skp2) complex through phosphorylated Thr187 in p27. Curr Biol 1999; 9: 661-664.

38. Herrup K, Yang Y. Cell cycle regulation in the postmitotic neuron: oxymoron or new biology? Nat Rev 2007; 8: 368-378.

39. Folch J, Junyent F, Verdaguer E, Auladell C, Pizarro JG, Beas-Zarate C et al. Role of cell cycle re-entry in neurons: a common apoptotic mechanism of neuronal cell death. Neurotox Res 2012; 22: 195-207.

40. Courchesne E, Pierce K, Schumann CM, Redcay E, Buckwalter JA, Kennedy DP et al. Mapping early brain development in autism. Neuron 2007; 56: 399-413.

41. Semendeferi K, Lu A, Schenker N, Damasio H. Humans and great apes share a large frontal cortex. Nat Neurosci 2002; 5: 272-276.

42. Peng $\mathrm{H}$, Huang $\mathrm{Y}$, Rose J, Erichsen D, Herek S, Fujii $\mathrm{N}$ et al. Stromal cell-derived factor 1-mediated CXCR4 signaling in rat and human cortical neural progenitor cells. J Neurosci Res 2004; 76: 35-50.

43. Garza JC, Guo M, Zhang W, Lu XY. Leptin increases adult hippocampal neurogenesis in vivo and in vitro. J Biol Chem 2008; 283: 18238-18247.

44. Yelamanchili SV, Chaudhuri AD, Chen LN, Xiong H, Fox HS. MicroRNA-21 dysregulates the expression of MEF2C in neurons in monkey and human SIV/HIV neurological disease. Cell Death Dis 2010; 1: e77.

45. Chaudhuri AD, Yelamanchili SV, Fox HS. Combined fluorescent in situ hybridization for detection of microRNAs and immunofluorescent labeling for cell-type markers. Front Cell Neurosci 2013; 7: 160.

46. Varallyay E, Burgyan J, Havelda Z. MicroRNA detection by northern blotting using locked nucleic acid probes. Nat Protoc 2008; 3: 190-196.

47. Tran N. Fast and simple micro-RNA northern blots. Biochem Insights 2009; 2: 1-3.

48. Al-Shahrour F, Diaz-Uriarte R, Dopazo J. FatiGO: a web tool for finding significant associations of Gene Ontology terms with groups of genes. Bioinformatics 2004; 20: $578-580$.

49. Schmid I, Sakamoto KM. Analysis of DNA content and green fluorescent protein expression. Curr Protoc Cytom 2001; Chapter 7: Unit 7.16.

50. Yelamanchili SV, Chaudhuri AD, Flynn CT, Fox HS. Upregulation of cathepsin D in the caudate nucleus of primates with experimental parkinsonism. Mol Neurodegener 2011; 6: 52 .

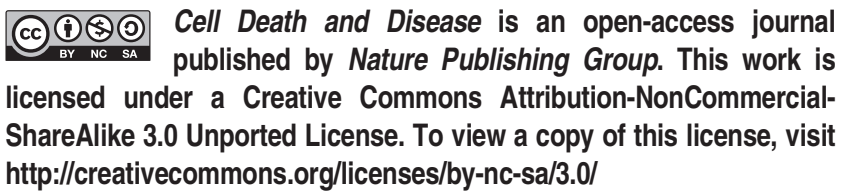

